\title{
Adaptive sliding-mode-backstepping trajectory tracking control of
}

\section{underactuated airships}

\author{
Shi Qian Liu ${ }^{\mathrm{a}}{ }^{\star}$, Yuan Jun Sang ${ }^{\mathrm{a}}$ James F. Whiborne \\ ${ }^{a}$ School of Aeronautics and Astronautics, Shanghai Jiao Tong University, Shanghai 200240, P.R.China \\ ${ }^{b}$ School of Aerospace, Transport and Manufacturing, Cranfield University, Cranfield MK43 0AL, UK
}

\begin{abstract}
The problem of trajectory tracking control for an underactuated stratospheric airship with model parameter uncertainties and wind disturbances is addressed in the paper. An adaptive backstepping sliding-mode controller is designed from the airship nonlinear dynamics model. The proposed controller has a two-level structure for trajectory guidance, tracking and stability, and the developed controller, based on nonlinear adaptive sliding-mode backstepping method, provides airship attitude and velocity control for the entire flight process. Furthermore, an active set based weighted least square algorithm is applied to find the optimal control surface inputs and the thruster commands under constraints of actuator saturation. The closed-loop system of trajectory tracking control plant is proved to be globally asymptotically stable by using Lyapunov theory. By comparing with traditional backstepping control and PID design, the results obtained demonstrate the capacity of the airship to execute a realistic trajectory tracking mission under two cases of lateral- and roll- underactuations, even in the presence of aerodynamic coefficient uncertainties, and wind disturbances.
\end{abstract}

Keywords: underactuated stratospheric airship system; sliding mode control; adaptive estimator; backstepping control; trajectory tracking

\footnotetext{
* Correspondence to: Shiqian Liu, School of Aeronautics and Astronautics, Shanghai Jiao Tong University, Shanghai, P. R. China, 200240 Academic visitor, Center for Aeronautics, Cranfield University, UK, MK43 0AL

† E-mail: liushiqian@sjitu.edu.cn
} 


\section{Introduction}

Stratospheric airships, like other unmanned aerial vehicles (UAVs), have potential military and civilian applications as observation, remote sensing and communication relay platforms. However, the airship dynamics is inherently nonlinear and distinct from common aircraft dynamics. The center of gravity (CG) of the airship is usually beneath the center of volume (CV), which is easily affected by air pressure and temperature, and the apparent mass and inertia of the airship are not constant. So it's rather complicated to design flight control for the stratospheric airship. Traditional control design approaches such as gain scheduling are hard to accomplish [1-3], because they are valid only in a small neighborhood of the equilibrium state. To overcome the theoretical limitations of linear control methods, many nonlinear control approaches have been studied for airship dynamics. Moutinho et al. proposed a dynamic inversion method to control the AURORA airship [4]. This approach cancels out the system nonlinearities completely, enables linear control design methods to be applied to the resulted feedback linearized system. However, there are some shortcomings such as over dependence on precise models for design. If the model has uncertainty, then robustness is not guaranteed.

To apply some useful nonlinear information, the backstepping approach is proposed to design robust controller for strict feedback systems through construction of Control Lyapunov functions (CLFs). Nonlinear backstepping design has been widely studied for other aircraft [5-6]. The backstepping method was first applied to process the flight decomposition control problem of an unmanned airship model [7]. A 50-meter-length mid-altitude unmanned airship was controlled by using a vectorial backstepping controller [8]. However, these designs are based on fully-actuated airship models, which cannot assure the performance for underactuated cases.

In this paper the airship is studied as an underactuated vehicle, mostly due to absence of a lateral force actuator to oppose aerodynamic side forces, to the limited roll moments available, and to the reduced authority of the control surfaces at low airspeeds [9-10]. Compared to fully actuated systems, it is more 
challenging to control underactuated systems because at least one degree of freedom cannot be directly controlled with an independent control input. Therefore, the underactuated airship control problem has been an active topic recently. Trajectory tracking, path following and hover stabilization controllers have been studied for underactuated vehicles via fuzzy control [11], robust gain scheduling [12], robust model predictive control [13] and some backstepping approaches [14-15]. Recently a controller without model information was proposed by using an expert demonstrations and a reinforcement learning method [16]. However, although the airship is underactuated, the vehicle has multiple effectors acting on the directly controlled degrees of freedom. This necessitates the use of control allocation. The above methods do not consider this.

Azinheira et al. proposed backstepping control designs for hover stabilization and path-tracking of a nonlinear underactuated airship model [9-10]. But their control allocation approach was based on the given relation between actuators and force inputs without given any optimization objective. Liesk et al. proposed a waypoint tracking controller for an unmanned finless airship [17]. However, the quadratic optimization control allocation algorithm in Liesk et al. requires an intensive computational load [17]. Liu et al. developed a vectorial backstepping method with active set control allocation to deal with saturation [18]. Yang et al. proposed a neural network approximation-based sliding-mode approach for positioning control of an autonomous airship[19]. However, these methods depend on the accurate model and are hard to deal with highly nonlinear airship dynamics with aerodynamic model uncertainties.

Motivated by the work of Liesk et al., Liu et al. and Yang et al. [17-19], this paper mainly concerns trajectory tracking control for the underactuated stratospheric airship, and the main contributions are as follows. A novel adaptive sliding-mode backstepping trajectory tracking controller (ASMB-TTC) is proposed for an underactuated stratospheric airship. The ASMB-TTC has two-level control structure including trajectory guider and the attitude and velocity controller. The ASMB-TTC has been applied to process two cases of underactuation for the airship in the lateral and roll motions. An active set based 
weighted least squares control allocation is embedded into the ASMB to find the optimal control inputs under underactuation and constraints of actuator saturation. The adaptive observers are designed for aerodynamic coefficient uncertainty and variable wind disturbances. Simulation results show the proposed ASMB -TTC has better performances in the trajectory tracking control for an underactuated airship in comparison with Azinheira's backstepping method and PID control.

This paper is organized as follows. Section 2 gives the nonlinear dynamics model of the underactuated stratospheric airship and presents the trajectory tracking problem. Section 3 proposes an ASMB-TTC design, and stability is analyzed for the associated closed-loop tracking error system. Simulations and performances of two underactuated cases with the ASMB-TTC are demonstrated in Section4. Section 5 gives some conclusions.

\section{Dynamics modeling and problem formulation}

The studied stratospheric airship is shown as Fig.1. There are two rudders and two elevators in the tail fins of the airship, two vectored propellers on both sides of the hull, and another on the stern for hovering control. The gondola is beneath the airship envelope to house the flight control system and other payloads.
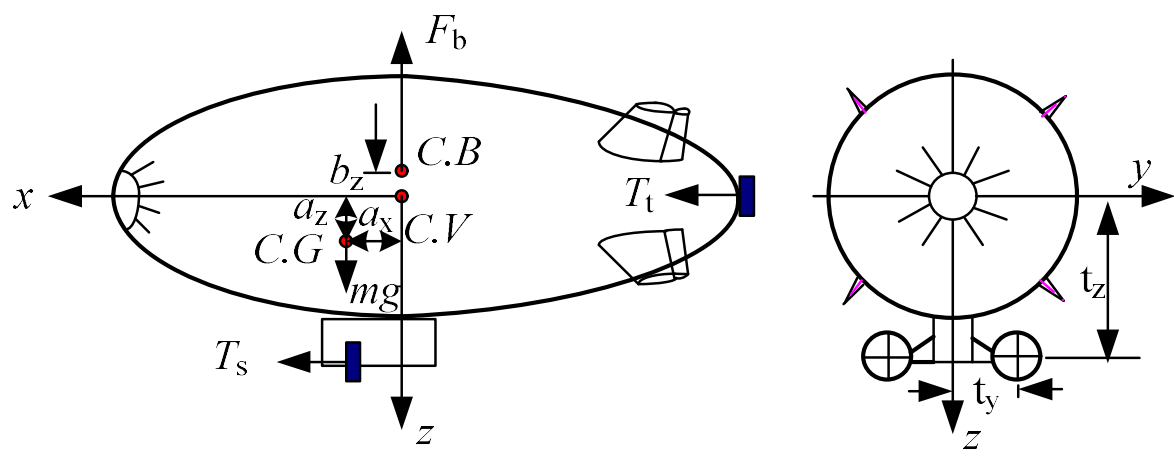

Fig. 1. structure of the stratospheric airship

\subsection{Airship Kinematics Model}

The kinematics model of the airship's position and attitude are as follows [12],[18],

$$
\begin{gathered}
\dot{\xi}=\boldsymbol{R}(\eta) v_{\mathrm{g}}=\boldsymbol{R}(\eta) v_{a}+v_{w}, \\
\dot{\eta}=\boldsymbol{J}(\eta) \omega,
\end{gathered}
$$


where $\xi=\left[\begin{array}{lll}x & y & z\end{array}\right]^{T}, \eta=\left[\begin{array}{lll}\phi & \theta & \psi\end{array}\right]^{T}, v_{a}=\left[\begin{array}{lll}u & v & w\end{array}\right]^{T}, \omega=\left[\begin{array}{lll}p & q & r\end{array}\right]^{T}, \boldsymbol{v}_{w}$ denotes the wind speed vector, and the wind field is assumed to be constant due to the stable meteorological condition in the stratosphere,

$$
v_{w}=\left[\begin{array}{c}
V_{w} c\left(\chi_{w}\right) c\left(\gamma_{w}\right) \\
V_{w} s\left(\chi_{w}\right) c\left(\gamma_{w}\right) \\
-V_{w} s\left(\gamma_{w}\right)
\end{array}\right]
$$

where $s($.$) and c($.$) denote sine and cosine functions respectively. V_{w}>0$ is the wind speed, $\gamma_{w}, \chi_{w}$ denote the horizontal and vertical wind direction angles. The direction cosine matrix $\boldsymbol{R}(\eta)$ and the rotational matrix $\boldsymbol{J}$ are as in Ref.[18].

\subsection{Airship Dynamics Model}

The airship dynamics is established as follows [20-24]:

$$
\boldsymbol{M}_{a}\left[\begin{array}{c}
\dot{v}_{a}(t) \\
\dot{\omega}(t)
\end{array}\right]=\left[\begin{array}{c}
\boldsymbol{f}_{k}+\boldsymbol{f}_{w}+\boldsymbol{f}_{G B}+\boldsymbol{f}_{A} \\
\boldsymbol{n}_{k}+\boldsymbol{n}_{w}+\boldsymbol{n}_{G B}+\boldsymbol{n}_{A}
\end{array}\right]+\left[\begin{array}{c}
\boldsymbol{f}_{C P} \\
\boldsymbol{n}_{C P}
\end{array}\right]
$$

where $\boldsymbol{M}_{a}$ denotes the generalized mass matrix as in [18]. Let $U=\left[\begin{array}{ll}\boldsymbol{f}_{C P}^{T} & \boldsymbol{n}_{C P}^{T}\end{array}\right]^{T}, \boldsymbol{x}=\left[\begin{array}{ll}v_{a}^{T} & \omega^{T}\end{array}\right]^{T}$, then (3) can be written as

$$
\dot{\boldsymbol{x}}(t)=\boldsymbol{f}(\boldsymbol{x})+\boldsymbol{g}(\boldsymbol{x}) U(t),
$$

where

$$
\boldsymbol{f}(\boldsymbol{x})=\boldsymbol{M}_{a}^{-1} \cdot\left[\begin{array}{l}
\boldsymbol{f}_{k}+\boldsymbol{f}_{w}+\boldsymbol{f}_{G B}+\boldsymbol{f}_{A} \\
\boldsymbol{n}_{k}+\boldsymbol{n}_{w}+\boldsymbol{n}_{G B}+\boldsymbol{n}_{A}
\end{array}\right]=\left[\begin{array}{c}
\left(\boldsymbol{f}_{V}\right)_{3 \times 1} \\
\left(\boldsymbol{f}_{\omega}\right)_{3 \times 1}
\end{array}\right], \boldsymbol{g}(\boldsymbol{x})=\boldsymbol{M}_{a}^{-1}=\left[\begin{array}{cc}
\left(\boldsymbol{g}_{V}\right)_{3 \times 3} & \left(\boldsymbol{g}_{V \omega}\right)_{3 \times 3} \\
\left(\boldsymbol{g}_{\omega V}\right)_{3 \times 3} & \left(\boldsymbol{g}_{\omega}\right)_{3 \times 3}
\end{array}\right] .
$$

$\boldsymbol{g}_{V}, \boldsymbol{g}_{V \omega}, \boldsymbol{g}_{\omega V}$ and $\boldsymbol{g}_{\omega}$ denote the sub-matrices of $\boldsymbol{M}_{a}^{-1}$,

$$
\boldsymbol{f}_{V}=\left[\begin{array}{ll}
\boldsymbol{g}_{V} & \boldsymbol{g}_{V \omega}
\end{array}\right] \cdot\left[\begin{array}{l}
\boldsymbol{f}_{k}+\boldsymbol{f}_{w}+\boldsymbol{f}_{G B}+\boldsymbol{f}_{A} \\
\boldsymbol{n}_{k}+\boldsymbol{n}_{w}+\boldsymbol{n}_{G B}+\boldsymbol{n}_{A}
\end{array}\right], \boldsymbol{f}_{\omega}=\left[\begin{array}{ll}
\boldsymbol{g}_{\omega V} & \boldsymbol{g}_{\omega}
\end{array}\right] \cdot\left[\begin{array}{l}
\boldsymbol{f}_{k}+\boldsymbol{f}_{w}+\boldsymbol{f}_{G B}+\boldsymbol{f}_{A} \\
\boldsymbol{n}_{k}+\boldsymbol{n}_{w}+\boldsymbol{n}_{G B}+\boldsymbol{n}_{A}
\end{array}\right] .
$$

The airship aerodynamic forces and moments on the right hand side of (3) can be expressed as follows [22, 
24],

$$
\left\{\begin{array}{l}
\boldsymbol{f}_{A i}=\bar{q} S_{r e f} C_{i} \\
\boldsymbol{n}_{A i}=\bar{q} S_{r e f} l_{r e f} C_{m i}
\end{array}(i=x, y, z),\right.
$$

where $C_{i}$ and $C_{m i}$ denote aerodynamic coefficients of forces and moments along $x$-,y-, and $z$-axes in $\boldsymbol{F}_{\mathrm{b}} . S_{\text {ref }}$ and $l_{r e f}$ denote the reference area and the reference length of the airship.

The kinematics force vector of the airship in (3) is

$$
\begin{gathered}
\boldsymbol{f}_{k}=\left[\begin{array}{c}
-m_{z} w q+m_{y} v r-m z_{G} p r+m x_{G}\left(r^{2}+q^{2}\right) \\
-m_{x} u r+m_{z} w p-m z_{G} q r-m x_{G} p q \\
-m_{y} v p+m_{x} u q+m z_{G}\left(p^{2}+q^{2}\right)-m x_{G} p r
\end{array}\right], \\
\boldsymbol{n}_{k}=\left[\begin{array}{c}
\left(J_{y}-J_{z}\right) q r+J_{x z} p q+m z_{G}(r u-p w) \\
\left(J_{z}-J_{x}\right) p r+J_{x z}\left(r^{2}-p^{2}\right)-m z_{G}(q w-r v)+m x_{G}(p v-q u) \\
\left(J_{x}-J_{y}\right) q p-J_{x z} q r-m x_{G}(r u-p w)
\end{array}\right] .
\end{gathered}
$$

The wind-induced force vector of the airship is

$$
\begin{aligned}
& \boldsymbol{f}_{w}=M_{B a} v_{w}+\omega \times M_{B a} v_{w}, \\
& \boldsymbol{n}_{w}=J_{B a} \dot{\omega}_{w}+\omega \times J_{B a} \omega_{w}
\end{aligned}
$$

where $v_{w}$ and $\omega_{w}$ denote the wind linear speed and angular rate vectors. Assuming the wind field constant in the stratosphere, the matrix $M_{B a}$ and $J_{B a}$ of apparent buoyancy mass and its inertial moment are

$$
M_{B a}=M_{v}+m_{B} I_{3}, J_{B a}=J_{v}+J_{B}
$$

where $m_{B}$ denotes buoyancy mass meeting $m_{B}=\rho \nabla$, and $J_{B} \in R^{3}$ is the inertial matrix of the buoyancy air. $M_{v}$ denotes virtual mass matrix subject to $M_{v}=\operatorname{diag}\left(-X_{\dot{u}},-Y_{\dot{v}},-Z_{\dot{w}}\right), J_{v}$ denotes virtual inertial matrix subject to $J_{v}=\operatorname{diag}\left(L_{\dot{p}}, M_{\dot{q}}, N_{\dot{r}}\right), X_{\dot{u}}, Y_{\dot{v}}, Z_{\dot{w}}, L_{\dot{p}}, M_{\dot{q}}, N_{\dot{r}}$ are Lamb's virtual mass and inertia terms, which are all proportional to the buoyancy mass $m_{\mathrm{B}}$.

The wind-induced forces and moments are [25] 


$$
\begin{gathered}
\boldsymbol{f}_{w}=\left(-X_{\dot{u}}+Y_{\dot{v}}\right)\left[\begin{array}{ccc}
0 & r & -q \\
r & 0 & 0 \\
-q & 0 & 0
\end{array}\right]\left[\begin{array}{c}
u_{w} \\
v_{w} \\
w_{w}
\end{array}\right]=\left(-X_{\dot{u}}+Y_{\dot{v}}\right)\left[\begin{array}{c}
v_{w} r-w_{w} q \\
u_{w} r \\
-u_{w} q
\end{array}\right], \\
\boldsymbol{n}_{w}=0 .
\end{gathered}
$$

The sum force of gravity and buoyancy of (3) in the body-fixed frame can be described as

$$
\left[\begin{array}{c}
\mathbf{f}_{G B} \\
\mathbf{n}_{G B}
\end{array}\right]=\left[\begin{array}{c}
f_{G B x} \\
f_{G B y} \\
f_{G B z} \\
n_{G B x} \\
n_{G B y} \\
n_{G B z}
\end{array}\right]=\left[\begin{array}{c}
(G-B) \cdot \mathbf{R}(3,1) \\
(G-B) \cdot \mathbf{R}(3,2) \\
(G-B) \cdot \mathbf{R}(3,3) \\
-G \cdot z_{G} \cdot \mathbf{R}(3,2) \\
G \cdot z_{G} \cdot \mathbf{R}(3,1)-G \cdot x_{G} \cdot \mathbf{R}(3,3) \\
G \cdot x_{G} \cdot \mathbf{R}(3,2)
\end{array}\right]
$$

where $\mathbf{R}(i, j)$ denotes the element in the $i^{\text {th }}$ line and the $j^{\text {th }}$ column of the direction cosine matrix $\mathbf{R}(\eta)$.

The thrust on the stern is not considered in flight, which is mainly used for hovering. For simplicity, two tilt angles and thrusts of the vectored propellers are assumed to be equal, and the effect of rudders on the side force is also ignored. The virtual control force in (3) is described as following

$$
\boldsymbol{U}(t)=\left[\begin{array}{l}
\mathbf{f}_{C P} \\
\mathbf{n}_{C P}
\end{array}\right]=\mathbf{B} \boldsymbol{u}(t)
$$

where $\boldsymbol{B}$ is the control effectiveness matrix, $\boldsymbol{u}$ represents thrusts and control surface deflections. If the airship is underactuated in y-direction, or a lateral force actuator to oppose aerodynamic side forces is absent [9], then (16) can be rewritten as

$$
\left[\begin{array}{c}
f_{C P x} \\
f_{C P z} \\
n_{C P x} \\
n_{C P y} \\
n_{C P z}
\end{array}\right]=\left[\begin{array}{cccccc}
1 & 0 & 0 & 0 & 0 & 0 \\
0 & 1 & \bar{q} C_{z \delta_{e}} & \bar{q} C_{z \delta_{e}} & 0 & 0 \\
0 & 0 & \bar{q} C_{l \delta_{e}} & -\bar{q} C_{l \delta_{e}} & \bar{q} C_{l \delta_{r}} & -\bar{q} C_{l \delta_{r}} \\
d z & d x & \bar{q} C_{m \delta_{e}} & \bar{q} C_{m \delta_{e}} & 0 & 0 \\
0 & 0 & 0 & 0 & \bar{q} C_{n \delta_{r}} & \bar{q} C_{n \delta_{r}}
\end{array}\right]\left[\begin{array}{c}
T_{x} \\
T_{z} \\
\delta_{e L} \\
\delta_{e R} \\
\delta_{r U} \\
\delta_{r B}
\end{array}\right],
$$

where $C_{z \delta_{e}}, C_{l \delta_{e}}, C_{l \delta_{r}}, C_{m \delta_{e}}, C_{n \delta_{r}}$ are the aerodynamic coefficients of the control surfaces, $T_{x}$ and $T_{z}$ are the thrust components in $x_{\mathrm{b}}$ axis and $z_{\mathrm{b}}$ axis, respectively, $d_{x}$ and $d_{z}$ denote distances from the centre of volume (CV) to the propeller in the $x_{\mathrm{b}}$ - and $z_{\mathrm{b}}$ - axis. The thrusts in (17) can be linearized by the following transformation: 


$$
\left\{\begin{array}{l}
T_{x}=\left(T_{p}+T_{s}\right) \cos \mu \\
T_{z}=-\left(T_{p}+T_{s}\right) \sin \mu
\end{array}\right.
$$

Since the aerodynamic coefficients are varying with AOA and airspeed, which results in bounded model uncertainties in the dynamics system (4), thus the model uncertainty $d$ is introduced and the system of (4) can be modified as

$$
\dot{\boldsymbol{x}}(t)=\boldsymbol{f}(\boldsymbol{x})+\boldsymbol{g}(\boldsymbol{x}) U(t)+\boldsymbol{d}
$$

where $\boldsymbol{d}=\left[\begin{array}{ll}\boldsymbol{d}_{V}^{T} & \boldsymbol{d}_{\omega}^{T}\end{array}\right]^{T}=\left[\begin{array}{c}\bar{q} S_{r e f} \Delta C_{i} \\ \bar{q} S_{r e f} l_{r e f} \Delta C_{m i}\end{array}\right]$, and $\Delta C_{i}$ and $\Delta C_{m i}(i=x, y, z)$ denote uncertainties of the aerodynamic coefficients along $x-, y$-, and $z$-axes in $\boldsymbol{F}_{\mathrm{b}}$. Since the airship has large inertia and its motion is slow and sedate, then $d$ can be assumed to be slow-varying disturbance, which can be estimated on-line by using adaptive law. If $d$ is a fast-varying disturbance, an observer with its state augmented with disturbance acceleration can be applied.

In this paper two underactuated cases are considered as follows:

Case 1. The airship is underactuated in y-direction (that is, the lateral control force $f_{C P y}=0$ in Eq.(16)), thus sway velocity $v$ cannot be directly controlled. If cross-wind is present, then the airship can align into the wind through yaw motion and so reduce lateral forces requirement to a low and acceptable value, in which the limited lateral forces result from available damping forces and the body and fin drags can stabilize a steady lateral motion, thus the lateral force input will gradually decrease and disappear in stationary conditions.

Case 2. The airship works in Case 1 and without ailerons or differential actuators (i.e., $\delta_{e L}=\delta_{e R}, \delta_{r U}=\delta_{r B}$, and the roll control moment $n_{C P x}=0$ in Eq.(16)), thus sway velocity $v$ and bank angle $\phi$ cannot be directly controlled. In this case the disturbance of the roll moment resulted from wind can be attenuated by the airship roll damping, thus the roll moment input will gradually decrease and disappear in stationary conditions.

\subsection{The Trajectory Tracking Control Problem}

Consider the airship models of (1.a), (1.b) and (19). Let $\xi_{r}(t):[0, \infty) \rightarrow \mathbb{R}^{3}$ be a given sufficiently smooth 
time-varying reference trajectory with its time-derivatives $\left(\dot{\xi}_{r}(t), \ddot{\xi}_{r}(t)\right)$ bounded. The control task is to design a trajectory tracking controller such that the closed-loop system meets following requirements:

1) The trajectory tracking error dynamics are globally asymptotically stable under specified model uncertainty and external disturbances, where the trajectory tracking error $\boldsymbol{e}(t)=\xi(t)-\xi_{r}(t)$;

2) The output trajectory $\xi$ is steered towards a given reference trajectory $\xi_{r}$ with $\lim _{t \rightarrow \infty}\|\boldsymbol{e}(t)\|<\varepsilon_{0}$, where $\varepsilon_{0}$ is a prescribed constant, 2-norm $\|\boldsymbol{e}(t)\|=\boldsymbol{e}^{T} \boldsymbol{e}$.

\section{$3 \quad$ ASMB trajectory tracking control design}

This section gives the overview of the ASMB-TTC for the under-actuated airship. The controller structure is proposed as Fig.2, where the trajectory guider provides attitude and velocity commands for the speed and attitude controller; while the inner-loop controller can be treated as an autopilot to stabilize the airship attitude and track the desired velocity. The structure of the ASMB-TTC design includes an adaptive sliding mode backstepping based attitude controller, a CLF based velocity controller, a virtual control calculation part and an active set control allocation module. The controller design is presented in detail in the next section.

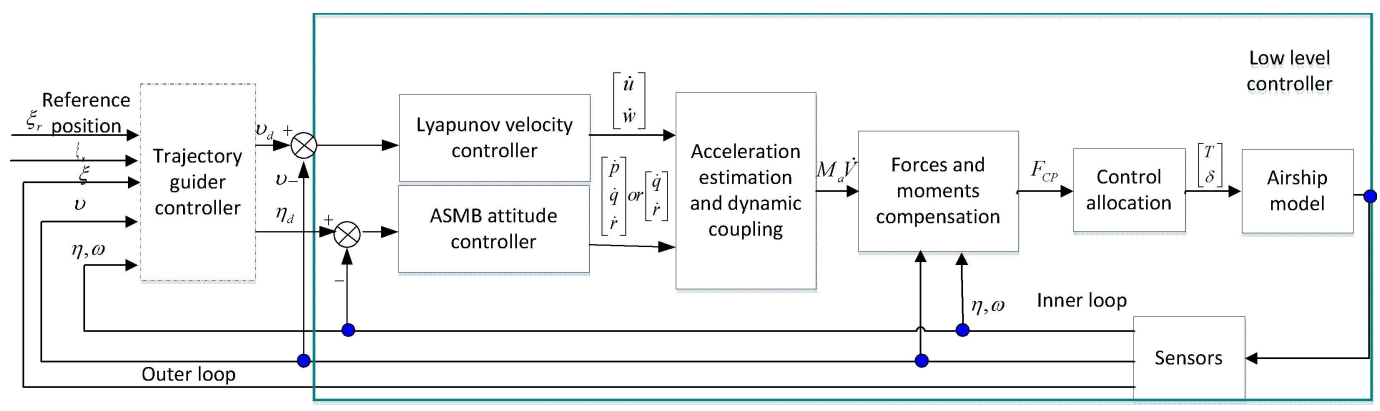

Fig. 2. Block diagram of the ASMB-TTC architecture

\subsection{Adaptive Sliding Mode Backstepping Based Attitude Controller}

In this section an adaptive sliding mode backstepping controller is designed; the objective is to make the attitude output $\boldsymbol{\eta}$ converge to the desired value vector $\boldsymbol{\eta}_{\boldsymbol{d}}$. First a SMC control is introduced to improve system 
adaptiveness. Since the general SMC control law is not designed to directly meet some desired closed-loop system performance, but to ensure the sliding surface is reached and motion on sliding mode surface is maintained, the so called reachability condition must be satisfied. The reachability condition means the trajectory of the system states must always point towards the sliding surface. In the case of single input system, it follows from Lyapunov method that

$$
\boldsymbol{s} \dot{\boldsymbol{s}}<0
$$

around $\boldsymbol{s}(t)=\mathbf{0}$, where $\boldsymbol{s}$ denotes sliding mode surface. If a positive parameter or function $\varsigma$ is defined then global asymptotic stability for the dynamics of $s(t)$ will given by [26]

$$
\dot{\boldsymbol{s}}=-\varsigma \operatorname{sgn}(\boldsymbol{s})
$$

where sgn(.) is sign function. A more strict reachability condition that ensures the sliding surface is reached despite the presence of uncertainty and in finite time, so the sliding surface is chosen as follows [27]

$$
\dot{\boldsymbol{s}}=\boldsymbol{u}_{S M C}=-h \boldsymbol{s}-\varsigma \operatorname{sgn}(\boldsymbol{s}),
$$

where $h, \varsigma$ are sliding mode surface parameters with $h>0, \varsigma>0$.

Now consider the kinematics model (1.a), (1.b) and the dynamics model (19). The attitude controller is derived in two steps.

Step 1 (Backstepping for the variation of $z_{1}$ ). The tracking error vector of attitudes is defined as

$$
\boldsymbol{z}_{1} \triangleq \boldsymbol{\eta}-\boldsymbol{\eta}_{d}
$$

and its derivative is

$$
\dot{\boldsymbol{z}}_{1} \triangleq \dot{\boldsymbol{\eta}}-\dot{\boldsymbol{\eta}}_{d}=J \omega-\dot{\boldsymbol{\eta}}_{d}
$$

Now let us define the Lyapunov function $V_{1}$, which is required to be positive definite around the desired position as follows:

$$
V_{1}=\frac{1}{2} z_{1}^{T} z_{1}
$$

Since the desired behavior for $\dot{\boldsymbol{\eta}}$ can still be chosen and it can be considered as the "virtual" control input. In 
the backstepping design this desired dynamic behavior is called the stabilizing function. Define the stabilizing function $\alpha$, as the virtual angular rate vector of $\dot{\boldsymbol{\eta}}_{r}$,

$$
\alpha \triangleq \dot{\boldsymbol{\eta}}_{d}-K_{1} \boldsymbol{z}_{1}
$$

where $\dot{\eta}_{r}$ represents the reference or virtual angular rate vector, $\boldsymbol{K}_{1}>0$ is often chosen as a diagonal matrix to simplify the design, i.e., $\boldsymbol{K}_{1}=\operatorname{diag}\left(k_{11}, k_{12}, k_{13}\right), k_{1 i}(i=1,2,3)$ is constant value.

Step 2 (Backstepping for the variation of $z_{2}$ ). Because the angular rate $\dot{\eta}$ is not a control input, there exists a dynamic error between it and its desired behavior of $\dot{\eta}_{r}$. To compensate this dynamic error, the speed tracking error vector for the attitude dynamics is defined as

$$
\boldsymbol{z}_{2} \triangleq \dot{\boldsymbol{\eta}}-\dot{\boldsymbol{\eta}}_{r}=J \omega-\alpha
$$

By using (3) and (19) the angular acceleration can be simplified as

$$
\dot{\boldsymbol{\omega}}=\ddot{\boldsymbol{\eta}} \approx \boldsymbol{f}_{\omega}+\boldsymbol{g}_{\omega} \boldsymbol{n}_{C P}+\boldsymbol{d}_{\omega}
$$

where $\boldsymbol{d}_{\omega}$ denotes the uncertainty of attitude dynamics, mainly including aerodynamic coefficient uncertainties.

Substituting (1.b) with consideration of attitude disturbance $\boldsymbol{d}_{\omega}$ yields the derivative of $\boldsymbol{z}_{2}$ as follows

$$
\dot{\boldsymbol{z}}_{2} \triangleq \ddot{\boldsymbol{\eta}}-\ddot{\boldsymbol{\eta}}_{r}=\frac{\mathrm{d}(J \boldsymbol{\omega})}{\mathrm{d} t}+\boldsymbol{d}_{\omega}-\frac{\mathrm{d} \alpha}{\mathrm{d} t}=\dot{J} \boldsymbol{\omega}+J \dot{\boldsymbol{\omega}}-\dot{\alpha}+\boldsymbol{d}_{\omega}
$$

Substituting (26) and (27) into (24) yields

$$
\dot{\boldsymbol{z}}_{1}=\boldsymbol{z}_{2}+\alpha-\dot{\boldsymbol{\eta}}_{d}=\boldsymbol{z}_{2}-K_{1} \boldsymbol{z}_{1}
$$

that is,

$$
\boldsymbol{z}_{2}=\dot{\boldsymbol{z}}_{1}+K_{1} \boldsymbol{z}_{1}
$$

Differentiating (31) and substituting (23) yields

$$
\dot{\boldsymbol{z}}_{2}=\ddot{\boldsymbol{\eta}}-\ddot{\boldsymbol{\eta}}_{d}+K_{1} \dot{\boldsymbol{z}}_{1}
$$

From (26) and (30), the derivative of the stabilizing function $\alpha$ is 


$$
\dot{\alpha}=\ddot{\boldsymbol{\eta}}_{d}-K_{1}\left(\boldsymbol{z}_{2}-K_{1} \boldsymbol{z}_{1}\right)=\ddot{\boldsymbol{\eta}}_{d}-K_{1} \boldsymbol{z}_{2}+K_{1}^{2} \boldsymbol{z}_{1}
$$

The derivative of the first CLF of (25) can be rewritten as follows by substituting (30)

$$
\dot{V}_{1}=\boldsymbol{z}_{1}^{T} \dot{z}_{1}=\boldsymbol{z}_{1}^{T}\left(\boldsymbol{z}_{2}-K_{1} \boldsymbol{z}_{1}\right)=-\boldsymbol{z}_{1}^{T} K_{1} \boldsymbol{z}_{1}+\boldsymbol{z}_{1}^{T} \boldsymbol{z}_{2}
$$

Now following the conventional linear sliding surface design method, the sliding surface $s$ is defined as follows

$$
s=\lambda_{1} z_{1}+z_{2},
$$

where the SMC parameter $\lambda_{1}=\operatorname{diag}\left(\lambda_{11}, \lambda_{12}, \lambda_{13}\right)$ with $\lambda_{1 \mathrm{i}}>0(i=1,2,3)$, and the derivative of sliding mode surface $s$ is

$$
\dot{\boldsymbol{s}}=\lambda_{1} \dot{\boldsymbol{z}}_{1}+\dot{\boldsymbol{z}}_{2}
$$

The second augmented CLF is constructed as

$$
V_{2}=V_{1}+\frac{1}{2} \boldsymbol{s}^{T} \boldsymbol{s}+\frac{1}{2 \gamma_{d}} \tilde{\boldsymbol{d}}_{\omega}^{T} \tilde{\boldsymbol{d}}_{\omega},
$$

where $\gamma_{d}$ is a positive constant that determines the convergence speed of the estimate. Differentiating (37) yields

$$
\dot{V}_{2}=\boldsymbol{z}_{1}^{T} \dot{\boldsymbol{z}}_{1}+\boldsymbol{s}^{T} \dot{\boldsymbol{s}}+\frac{1}{\gamma_{d}} \tilde{\boldsymbol{d}}_{\omega}^{T} \dot{\tilde{\boldsymbol{d}}}_{\omega}
$$

Since $\boldsymbol{d}_{\omega}$ is an unknown constant or slow-varying disturbance, the derivative of the estimated parameter error is

$$
\dot{\tilde{\boldsymbol{d}}}_{\omega}=\dot{\hat{\boldsymbol{d}}}_{\omega}-\dot{\boldsymbol{d}}_{\omega}=\dot{\hat{\boldsymbol{d}}}_{\omega}
$$

where $\hat{d}_{\omega}$ and $\tilde{d}_{\omega}$ are the estimated parameter vector of $d_{\omega}$ and the associated estimated parameter error vector. Substituting (29), (30), (32), (34), (35) and (39) into (38) yields

$$
\dot{V}_{2}=\boldsymbol{z}_{1}^{T} \dot{\boldsymbol{z}}_{1}+\boldsymbol{s}^{T} \dot{\boldsymbol{s}}+\frac{1}{\gamma_{d}} \tilde{\boldsymbol{d}}_{\omega}^{T} \dot{\tilde{\boldsymbol{d}}}_{\omega}
$$




$$
\begin{aligned}
& =-\boldsymbol{z}_{1}^{T} K_{1} \boldsymbol{z}_{1}+\boldsymbol{z}_{1}^{T} \boldsymbol{z}_{2}+\boldsymbol{s}^{T}\left(\lambda_{1} \dot{\boldsymbol{z}}_{1}+\dot{\boldsymbol{z}}_{2}\right)+\frac{1}{\gamma_{d}} \tilde{\boldsymbol{d}}_{\omega}^{T} \dot{\hat{\boldsymbol{d}}}_{\omega} \\
& =-\boldsymbol{z}_{1}^{T} K_{1} \boldsymbol{z}_{1}+\boldsymbol{z}_{1}^{T} \boldsymbol{z}_{2}+\boldsymbol{s}^{T}\left(\lambda_{1}\left(\boldsymbol{z}_{2}-K_{1} \boldsymbol{z}_{1}\right)+\left(\ddot{\boldsymbol{\eta}}-\ddot{\boldsymbol{\eta}}_{d}+K_{1} \dot{\boldsymbol{z}}_{1}\right)\right)+\frac{1}{\gamma_{d}} \tilde{\boldsymbol{d}}_{\omega}^{T} \dot{\hat{\boldsymbol{d}}}_{\omega} \\
& =-\boldsymbol{z}_{1}^{T} K_{1} \boldsymbol{z}_{1}+\boldsymbol{z}_{1}^{T} \boldsymbol{z}_{2}+\boldsymbol{s}^{T}\left(\lambda_{1}\left(\boldsymbol{z}_{2}-K_{1} \boldsymbol{z}_{1}\right)+\dot{J} \boldsymbol{\omega}+J \dot{\boldsymbol{\omega}}+K_{1} \dot{\boldsymbol{z}}_{1}-\ddot{\eta}_{d}+\boldsymbol{d}_{\omega}\right)+\frac{1}{\gamma_{d}} \tilde{\boldsymbol{d}}_{\omega}^{T} \dot{\hat{\boldsymbol{d}}}_{\omega} .
\end{aligned}
$$

A desired control input is selected as follows to make (38) semi-negative definite,

$$
\begin{aligned}
\dot{\omega}_{d} & =J^{-1}\left(-\lambda_{1}\left(z_{2}-K_{1} z_{1}\right)-\dot{J} \omega+\ddot{\boldsymbol{\eta}}_{d}-K_{1} \dot{\boldsymbol{z}}_{1}-\hat{\boldsymbol{d}}_{\omega}-h(s+\varsigma \operatorname{sgn}(s))\right) \\
& =J^{-1}\left(-\left(\lambda_{1}+K_{1}\right) \dot{\boldsymbol{z}}_{1}-\dot{J} \boldsymbol{\omega}+\ddot{\boldsymbol{\eta}}_{d}-\hat{\boldsymbol{d}}_{\omega}-h(s+\varsigma \operatorname{sgn}(s))\right),
\end{aligned}
$$

where $h, \varsigma$ are sliding-mode parameters as in (22).

The sliding mode term in the backstepping design is to improve system adaptiveness to model uncertainties, even in the presence of the disturbances. Substituting (41) with $\boldsymbol{s}^{T} \tilde{\boldsymbol{d}}_{\omega}=\tilde{\boldsymbol{d}}_{\omega}^{T} \boldsymbol{s}$ into (40) yields

$$
\begin{aligned}
\dot{V}_{2}= & \left(-\boldsymbol{z}_{1}^{T} K_{1} \boldsymbol{z}_{1}+\boldsymbol{z}_{1}^{T} \boldsymbol{z}_{2}\right)-h \boldsymbol{s}^{T} \boldsymbol{s}-h \boldsymbol{s}|\boldsymbol{s}|+\boldsymbol{s}^{T}\left(\boldsymbol{d}_{\omega}-\hat{\boldsymbol{d}}_{\omega}\right)+\tilde{\boldsymbol{d}}_{\omega}^{T}\left(\frac{1}{\gamma_{d}} \dot{\hat{\boldsymbol{d}}}_{\omega}\right) \\
& =\left(-\boldsymbol{z}_{1}^{T} K_{1} \boldsymbol{z}_{1}+\boldsymbol{z}_{1}^{T} \boldsymbol{z}_{2}\right)-h \boldsymbol{s}^{2}-h \varsigma|\boldsymbol{s}|+\tilde{\boldsymbol{d}}_{\omega}^{T}\left(\frac{1}{\gamma_{d}} \dot{\hat{d}}_{\omega}-\boldsymbol{s}\right) .
\end{aligned}
$$

Choose the update law as:

$$
\dot{\hat{\boldsymbol{d}}}_{\omega}=\gamma_{d} \boldsymbol{s},
$$

and yields

$$
\dot{V}_{2}=\left(-z_{1}^{T} K_{1} z_{1}+z_{1}^{T} z_{2}\right)-h s^{2}-h \varsigma|s|
$$

Define $Q$ as a positive definite symmetric matrix

$$
Q \triangleq\left[\begin{array}{cc}
K_{1}+h \lambda_{1}^{2} & \lambda_{1} h-0.5 \boldsymbol{I}_{n} \\
\lambda_{1} h-0.5 \boldsymbol{I}_{n} & h \boldsymbol{I}_{n}
\end{array}\right]
$$

where $\boldsymbol{I}_{\mathrm{n}}$ denotes the $n \times n$ unit matrix. Let $Z_{12} \triangleq\left[\begin{array}{ll}\boldsymbol{z}_{1}^{T} & \boldsymbol{z}_{2}^{T}\end{array}\right]^{\mathrm{T}}$, this then yields 


$$
\begin{aligned}
Z_{12}^{T} Q Z_{12}= & \boldsymbol{z}_{1}^{T} K_{1} \boldsymbol{z}_{1}+h \lambda_{1}^{2} \boldsymbol{z}_{1}^{T} \boldsymbol{z}_{1}+2 \lambda_{1} h \boldsymbol{z}_{1}^{T} \boldsymbol{z}_{2}-\boldsymbol{z}_{1}^{T} \boldsymbol{z}_{2}+h \boldsymbol{z}_{2}^{T} \boldsymbol{z}_{2} \\
& =\boldsymbol{z}_{1}^{T} K_{1} \boldsymbol{z}_{1}-\boldsymbol{z}_{1}^{T} \boldsymbol{z}_{2}+h\left(\lambda_{1} \boldsymbol{z}_{1}+\boldsymbol{z}_{2}\right)^{T}\left(\lambda_{1} \boldsymbol{z}_{1}+\boldsymbol{z}_{2}\right) \\
& =\left(\boldsymbol{z}_{1}^{T} K_{1} \boldsymbol{z}_{1}-\boldsymbol{z}_{1}^{T} \boldsymbol{z}_{2}\right)+h \boldsymbol{s}^{T} \boldsymbol{s}
\end{aligned}
$$

Substituting (46) into (44) yields

$$
\dot{V}_{2}=-Z_{12}^{T} Q Z_{12}-h \varsigma|\boldsymbol{s}| .
$$

Using (45), it is obtained that

$$
\begin{aligned}
|Q|= & \left|\left[\begin{array}{cc}
K_{1}+h \lambda_{1}^{2} & \lambda_{1} h-0.5 \boldsymbol{I}_{n} \\
\lambda_{1} h-0.5 \boldsymbol{I}_{n} & h \boldsymbol{I}_{n}
\end{array}\right]\right| \\
& =h\left(K_{1}+\lambda_{1}\right)-(1 / 4) \boldsymbol{I}_{n} .
\end{aligned}
$$

If the following condition is satisfied,

$$
h\left(K_{1}+\lambda_{1}\right)>1 / 4 \boldsymbol{I}_{n},
$$

then $Q$ is positive definite, and if $h \varsigma>0$, then

$$
\dot{V}_{2}=-Z_{12}^{T} Q Z_{12}-h \varsigma|\boldsymbol{s}|<0
$$

Therefore, robust stability of the closed-loop system can be guaranteed by using the ASMB controller according to Lyapunov theory.

From above analysis, the structure of the adaptive integral backstepping controller for attitude control can be designed as Fig.3, which includes attitude dynamics of the airship and adaptive sliding mode backstepping control with an adaptive disturbance estimator. 


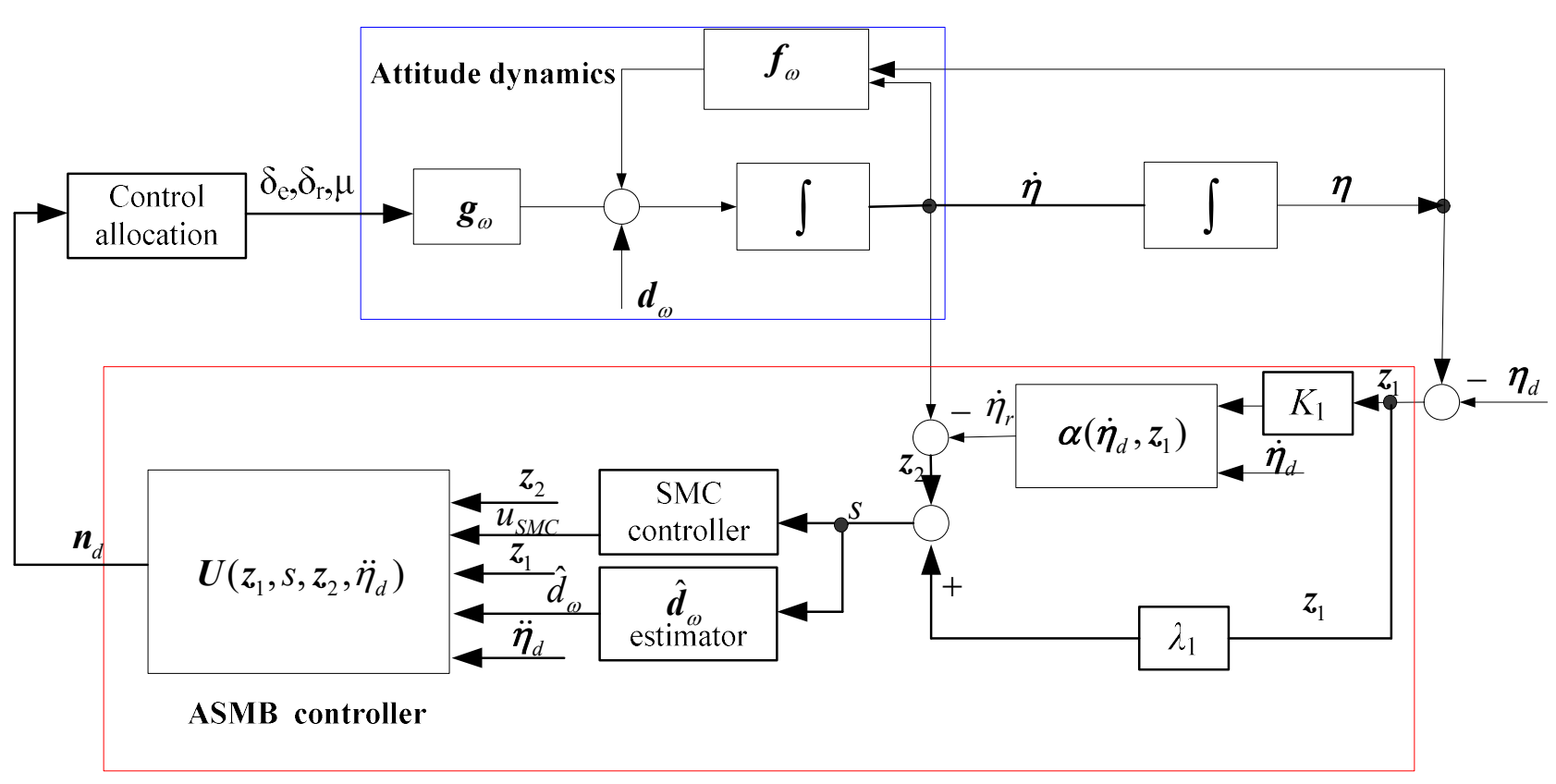

Fig.3 block of the adaptive sliding mode backstepping controller

So the desired control (41) can be implemented as following information flow:

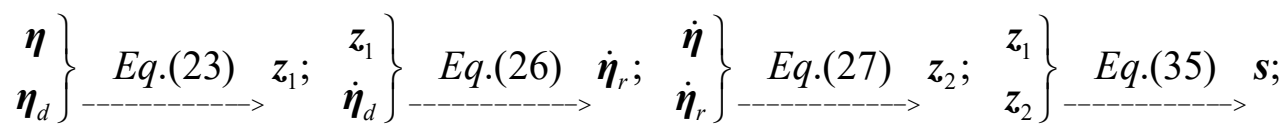

$$
\begin{aligned}
& \boldsymbol{s} \quad \text { Eq.(43) } \quad \dot{\hat{\boldsymbol{d}}}_{\omega} \rightarrow \int \dot{\hat{\boldsymbol{d}}}_{\omega} d t \rightarrow \hat{\boldsymbol{d}}_{\omega} ; \boldsymbol{s} \rightarrow \boldsymbol{u}_{s m c}=-h(\boldsymbol{s}+\varsigma \operatorname{sgn}(\boldsymbol{s})) ;
\end{aligned}
$$

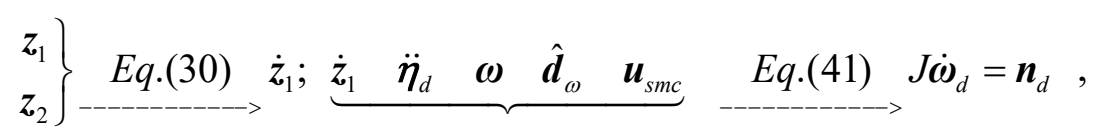

where $\boldsymbol{n}_{d}$ is the desired moment input.

Remark 1. It can be seen from the control input design in (41), $\lambda_{1}$ affects the transient response, $K_{1}$ has an influence when the system approaches the sliding surface, and $h$ affects the steady-state response. If these three parameters are chosen to be too large or too small, the system will have large overshoot and steady-state error or one have a slow rise time and settling time.

\subsection{Velocity Control Based on CLF}

This section is to design a backstepping velocity controller, and the objective is to make the airspeed $v_{a}$ of the airship converge to the desired values $v_{a, d}$. The velocity can be controlled directly via the acceleration of 
the airship. However, the vectored propellers are mounted such that they can generate forces in $x_{\mathrm{b}}-$ and $z_{\mathrm{b}}-$ directions but not in the lateral $y_{\mathrm{b}}$ - direction. This means that the velocity in the body $\mathrm{y}-$ direction cannot be controlled directly. The lateral underactuated problem of the airship is discussed here. Now the velocity tracking error vector is defined as

$$
\boldsymbol{z}_{3} \triangleq v_{a}-v_{d}
$$

where $v_{a}=\left[\begin{array}{ll}u & w\end{array}\right]^{T}$. And the associated CLF is chosen as

$$
V_{3}=\frac{1}{2} z_{3}^{T} z_{3}
$$

Differentiating (53) with respect to time yields

$$
\dot{V}_{3}=\boldsymbol{z}_{3}^{T} \dot{z}_{3}=\boldsymbol{z}_{3}^{T}\left(\dot{v}_{a}-\dot{v}_{d}\right) .
$$

In according to Lyapunov theory, $\dot{V}_{3}$ is required to be semi-negative definite (that is, $\dot{V}_{3}=-K_{3} z_{3}^{T} z_{3}$ ). To stabilize the closed-loop system, the desired linear acceleration vector can be obtained from (53) and (54):

$$
\dot{v}_{a, d}=\dot{v}_{d}-K_{3} z_{3}=\dot{v}_{d}+K_{3} v_{d}-K_{3} v_{a}
$$

where control gain matrices $K_{3}=\operatorname{diag}\left(k_{31}, k_{32}\right)>0 . k_{31}$ and $k_{32}$ are constant, $\dot{v}_{a, d}$ denotes desired linear acceleration vector in $\mathbf{F}_{\mathrm{b}}$.

Remark 2. The parameters $v_{a, d} \in \mathbb{R}^{2}, v_{d} \in \mathbb{R}^{2}$, and $K_{3} \in \mathbb{R}^{2 \times 2}$ (for Case 1 and Case 2).

$$
\eta \in \mathbb{R}^{2}, \eta_{d} \in \mathbb{R}^{2}, \omega \in \mathbb{R}^{2} \text {, and } K_{P} \in \mathbb{R}^{2 \times 2}, K_{D} \in \mathbb{R}^{2 \times 2}, J \in \mathbb{R}^{2 \times 2} \text { (for Case 2). }
$$

\subsection{Calculation of the Virtual Control}

This section is to achieve the virtual control inputs of the underactuated airship. The objective is to get the virtual control of $\dot{v}_{\mathrm{m}}$ and $\dot{p}_{\mathrm{m}}$ and form the total control force and moment of $\boldsymbol{U}$ for control allocation. To calculate the virtual control inputs under condition of underactuated cases accurately, the control law of acceleration must be modified. Two cases as in Section 2.2 are discussed here.

For Case 1, the lateral acceleration $\dot{v}$ in (55) cannot be directly applied. By using the motion relation 
described in (3), the lateral velocity of $\dot{v}$ can be achieved to make the desired control force in $\mathrm{y}_{\mathrm{b}}$-direction vanish in stationary conditions, that is,

$$
f_{C P y}=0,
$$

where the dynamics of the control force in $y_{b}$-direction is described as

$$
f_{C P y}=m_{y} \dot{v}-m z_{G} \dot{p}+m x_{G} \dot{r}-f_{k y}-f_{w y}-f_{G B y}-f_{A y} .
$$

Since $\dot{p}$ and $\dot{r}$ are given control laws in (41), the control law of lateral acceleration $\dot{v}$ is updated as follows

$$
\dot{v}_{m}=\frac{m}{m_{y}}\left(z_{G} \dot{p}-x_{G} \dot{r}+\frac{f_{k y}+f_{w y}+f_{G B y}+f_{A y}}{m}\right) .
$$

where $\mathrm{v}_{\mathrm{m}}$ is the control law integration of the lateral acceleration.

For Case 2, the roll acceleration $\dot{p}$ in (41) and the lateral acceleration $\dot{v}$ in (55) cannot be directly applied. So it should find values for $\dot{v}$ and $\dot{p}$ simultaneously which make the desired lateral force and roll moment vanish in stationary conditions, and it yields

$$
f_{C P y}=0, n_{C P x}=0,
$$

where the dynamics equations are as follows

$$
\left\{\begin{array}{l}
f_{C P y}=m_{y} \dot{v}-m z_{G} \dot{p}+m x_{G} \dot{r}-f_{k y}-f_{w y}-f_{G B y}-f_{A y} \\
n_{C P x}=-m z_{G} \dot{v}+J_{x} \dot{p}-J_{x z} \dot{r}-n_{k x}-n_{w x}-n_{G B x}-n_{A x}
\end{array} .\right.
$$

Since the yaw acceleration of $\dot{r}$ is a given control law in (41), the control laws of $\dot{v}$ and $\dot{p}$ can be updated as

$$
\begin{gathered}
\dot{v}_{m}=\frac{\left(J_{x} m x_{G}-J_{x z} m z_{G}\right) \dot{r}-J_{x}\left(f_{k y}+f_{w y}+f_{G B y}+f_{A y}\right)-m z_{G}\left(n_{k x}+n_{w x}+n_{G B x}+n_{A x}\right)}{m^{2} z_{G}{ }^{2}-J_{x} m_{y}}, \\
\dot{p}_{m}=\frac{\left(m^{2} z_{G} x_{G}-J_{x z} m_{y}\right) \dot{r}-m z_{G}\left(f_{k y}+f_{w y}+f_{G B y}+f_{A y}\right)-m_{y}\left(n_{k x}+n_{w x}+n_{G B x}+n_{A x}\right)}{m^{2} z_{G}{ }^{2}-J_{x} m_{y}},
\end{gathered}
$$

when all of the control laws of the desired linear accelerations and angular accelerations are obtained, the associated control forces and moments can be achieved by the following equation:

$$
\boldsymbol{U}=\boldsymbol{M}_{a} \dot{\boldsymbol{V}}-\boldsymbol{F}_{k}-\boldsymbol{F}_{w}-\boldsymbol{F}_{G B}-\boldsymbol{F}_{A},
$$


where $\dot{V}=\left[\dot{u}, \dot{v}_{m}, \dot{w}, \dot{p}, \dot{q}, \dot{r}\right]^{T}$ for Case 1 , and $\dot{V}=\left[\dot{u}, \dot{v}_{m}, \dot{w}, \dot{p}_{m}, \dot{q}, \dot{r}\right]^{T}$ for Case 2 .

To obtain the practical control surfaces and thruster inputs, a control allocation problem is presented to solve (16) with actuator saturation constraints:

$$
\boldsymbol{U}=\left[\begin{array}{l}
\mathbf{f}_{C P} \\
\mathbf{n}_{C P}
\end{array}\right]=\boldsymbol{B} \boldsymbol{u},
$$

subject to

$$
\max \left(\boldsymbol{u}(t-T)+\dot{\boldsymbol{u}}_{\min } T, \boldsymbol{u}_{\min }\right)=\underline{u} \leq \boldsymbol{u} \leq \bar{u}=\min \left(\boldsymbol{u}(t-T)+\dot{\boldsymbol{u}}_{\max } T, \boldsymbol{u}_{\max }\right),
$$

where $u_{\min }, u_{\max } \dot{\boldsymbol{u}}_{\min }$ and $\dot{\boldsymbol{u}}_{\max }$ denote lower and upper bounds of the actuator position and rotating rate, respectively, $\underline{u}$ and $\bar{u}$ are lower and upper bounds of the actuator inputs, $T$ denotes the sample period or motion period.

The control allocation problem of $(63) \sim(65)$ can be transformed into a constrained optimization problem by using the Active Set (AS) based Weighted Least Square (WLS) allocation principle [18]. Through the AS control allocation algorithm, the control allocation component can be implemented and incorporated into the ASMB-TTC scheme. When the optimal solution $\boldsymbol{u}^{*}$ is found, the practical control input signals, including thrust, tilt angle and control surface deflections, are obtained.

\subsection{The trajectory guider controller}

This section is to realize the trajectory guider, and the objective is to generate the desired attitude and velocity commands. For the trajectory guider controller, it has to calculate the desired attitudes and speeds in $x$ and $z$-direction such that the airship follows the desired path despite the inability to exert direct control over the speed in y-direction. According to the virtual spring damping principle, the desired or commanded velocity ${\dot{v_{c}}}_{c}$ in $\mathbf{F}_{g}$ is first obtained by the following differential equation as in the reference [28],

$$
\dot{v}_{c}=\sigma_{2}\left(v_{r}-v_{c}+\sigma_{1}\left(\boldsymbol{r}_{r}-\boldsymbol{r}_{g}\right)\right)+\dot{v}_{r}
$$

where $\sigma_{1}$ and $\sigma_{2}$ are the controller design parameters with $\sigma_{1}>0$ and $\sigma_{2}>0 . \sigma_{1} \cdot \sigma_{2}$ represents the spring 
stiffness coefficient and $\sigma_{2}$ denotes the damping coefficient, $\boldsymbol{r}_{\mathrm{g}}$ is the actual position $\boldsymbol{\xi}$ of the airship, $\boldsymbol{r}_{\mathrm{r}}$ is the desired position $\boldsymbol{\xi}_{d}, v_{g}$ is the actual airship velocity in $\mathbf{F}_{g}$. The airship desired attitude $\eta_{d}$ and velocity in $\mathbf{F}_{b}$ can be calculated by using the vehicle reference velocity $v_{r}$ and the current distance $\boldsymbol{r}_{D}$ between the airship and the reference vehicle, see Fig.4.

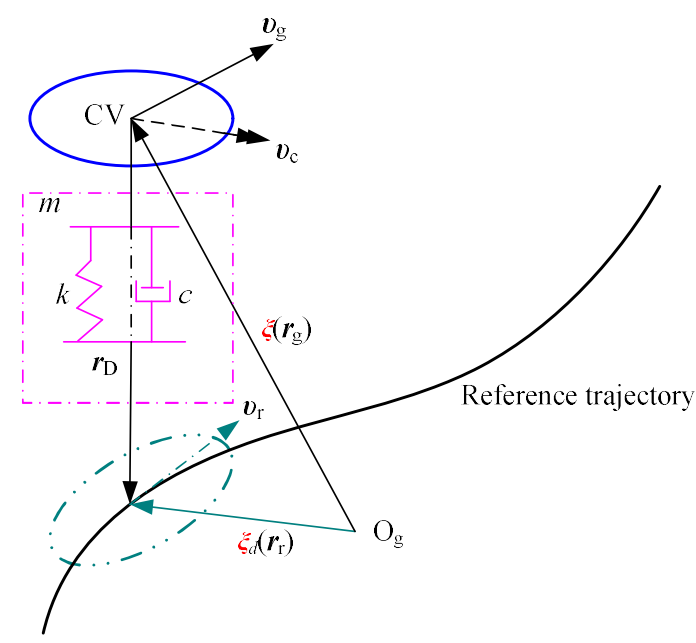

Fig.4 the virtual spring-damper system used for trajectory tracking control

It can be seen that perfect tracking without wind requires that $\dot{r}_{g}=v_{g}=v_{c}=\dot{\xi}$, and the desired attitude $\eta_{d}$ and velocity $v_{d}$ in the body-fixed frame are generated by using kinematics relation and the command velocity of $v_{c}$, the detailed design procedure of the trajectory guider can be seen from the reference [18], so this will not be described here.

Remark 3. For underactuated airships, the desired attitude $\eta_{d}$ and velocity $v_{d}$ generated by the trajectory guider controller are part of the whole vector, that is, for Case 1: $v_{d}=\left[u_{d b}, w_{d b}\right]^{\mathrm{T}}, \boldsymbol{\eta}_{d}=\left[\phi_{d}, \theta_{d}, \psi_{d}\right]^{\mathrm{T}}$; for Case 2: $v_{d}=\left[u_{d b}, w_{d b}\right]^{\mathrm{T}}, \boldsymbol{\eta}_{d}=\left[\begin{array}{ll}\theta_{d}, & \psi_{d}\end{array}\right]^{\mathrm{T}}$.

\subsection{Stability Analysis}

Lemma 1. [29]. Consider the nonlinear system $\dot{\boldsymbol{x}}(t)=f(\boldsymbol{x})$ with the equilibrium point $\boldsymbol{x}^{*}$. Let $V(x): \mathbb{C} \rightarrow \mathbb{R}$ be a continuously differentiable function such that for $\boldsymbol{x} \in \mathbb{C}\left(\mathbb{C} \subset \mathbb{R}^{n}\right)$ 
1) $V(\boldsymbol{x})>0$ (positive definite) with $\dot{V}(\boldsymbol{x})<0$ (negative definite),

2) $V(\boldsymbol{x}) \rightarrow \infty$ as $\|\boldsymbol{x}\|^{2} \rightarrow \infty$ (radially unbounded),

then the equilibrium point $\boldsymbol{x}=0$ satisfying $\mathrm{f}\left(\boldsymbol{x}^{*}\right)=0$ is globally asymptotically stable.

Assumption A1. The reference trajectory $\xi_{r}(t):[0, \infty) \rightarrow \mathbb{R}^{3}$ is sufficiently smooth with its time-derivatives $\left(v_{r}(t)=\dot{\xi}_{r}(t), \dot{v}_{r}(t)\right)$ bounded.

Because the trajectory tracking control is based on guidance, the control objective in the speed loop is that the guidance-based command signal $v_{c}(t)$ can be tracked by the system output of velocity $\dot{\xi}(t)$.

Assumption A2. The output signal $\dot{\xi}(t)$ or $\dot{r}_{g}(t)$ tracks the commanded signal $v_{c}(t)$ in (66) without steady tracking error, i.e., $\lim _{t \rightarrow \infty} \dot{\xi}(t)=\lim _{t \rightarrow \infty} v_{c}(t)$ with $\dot{\xi}(t)=R(\eta) v_{a}+v_{w}$.

Although wind speed $v_{w}$ can be regarded as one kind of external disturbances and adaptively estimated by the adaptive estimator as Eq.(43), it is hard to separate wind and model uncertainty. So a wind observer is introduced to improve tracking performances.

Choose a wind observer with the states $\left[\begin{array}{c}\hat{\xi} \\ \hat{v}_{w}\end{array}\right], \hat{\xi}$ and $\hat{v}_{w}$ denote estimation of $\xi$ and $v_{w}$, and design its dynamics as [10]

$$
\left[\begin{array}{c}
\dot{\hat{\xi}} \\
\dot{\hat{v}}_{w}
\end{array}\right]=\left[\begin{array}{c}
R(\eta) v_{a} \\
\mathbf{0}
\end{array}\right]+\left[\begin{array}{cc}
L_{\xi} & I_{3} \\
L_{w} & \mathbf{0}
\end{array}\right]\left[\begin{array}{c}
\xi-\hat{\xi} \\
\hat{v}_{w}
\end{array}\right]
$$

The estimation error can be obtained as

$$
\dot{\boldsymbol{e}}_{4}=\left[\begin{array}{c}
\xi-\hat{\xi} \\
\boldsymbol{v}_{w}-\hat{\boldsymbol{v}}_{w}
\end{array}\right]=\left[\begin{array}{cc}
L_{\xi} & I_{3} \\
L_{w} & \mathbf{0}
\end{array}\right] \boldsymbol{e}_{4}=\tilde{A}_{e} \boldsymbol{e}_{4}
$$

where $L_{\xi}, L_{w}$ are gain matrices subject to $\tilde{A}_{e}$ be Hurwitz. So there exists a positive definite symmetrical matrix $P_{e}$ such that 


$$
\frac{d}{d t}\left(\boldsymbol{e}_{4}^{T} P_{e} \boldsymbol{e}_{4}\right)=-\boldsymbol{e}_{4}^{T} Q_{e} \boldsymbol{e}_{4}
$$

where $Q_{e}=\operatorname{diag}\left(Q_{\xi}, Q_{v_{w}}\right), Q_{\xi}$ and $Q_{v_{w}}$ are symmetric positive definite matrices and the weight matrix $P_{e}$ satisfies the algebra Riccati equation,

$$
\tilde{A}_{e}^{T} P_{e}+P_{e} \tilde{A}_{e}=-Q_{e}
$$

Theorem 1. Consider the system of (1.a), (1.b) and (19) with Assumption A1 and A2 being satisfied. If there exist appropriately dimensioned diagonal matrices of $K_{1}, K_{3}, \lambda_{1}, L_{\xi}, L_{\mathrm{w}}$, constant parameters of $h, \varsigma, \gamma_{\mathrm{d}}$, and sufficiently small scalar parameter $\varepsilon$, such that $K_{1}>0, K_{3}>0, \lambda_{1}>0, h>0, \varsigma>0, \gamma_{\mathrm{d}}$ meeting with (43), $\varepsilon \leq \min \left\{\sqrt{\sigma_{1} \sigma_{2}}, 4 \sigma_{1} \sigma_{2} /\left(4 \sigma_{1}+\sigma_{2}\right)\right\}$, and $L_{\xi}, L_{w}$ are subject to $\tilde{A}_{e}$ being Hurwitz, then the ASMB-TTC, given by (41), (55) and (63), can guarantee the requirements of 1) and 2) in section 2.3.

\section{Proof.}

Define tracking error of position and velocity for the trajectory guider controller as $\boldsymbol{z}_{4} \triangleq\left(\begin{array}{c}\xi-\xi_{r} \\ v_{c}-v_{r}\end{array}\right)$. A Lyapunov function for the entire system is established in accordance with (37) and (53):

$$
V=V_{l}+V_{h}=\left(\frac{1}{2} z_{1}^{T} z_{1}+\frac{1}{2} \boldsymbol{s}^{T} \boldsymbol{s}+\frac{1}{2 \gamma_{d}} \tilde{\boldsymbol{d}}_{\omega}^{T} \tilde{\boldsymbol{d}}_{\omega}+\frac{1}{2} \boldsymbol{z}_{3}^{T} z_{3}\right)+\left(\frac{1}{2} \boldsymbol{z}_{4}^{T} P \boldsymbol{z}_{4}+\frac{1}{2} \boldsymbol{e}_{4}^{T} P_{e} \boldsymbol{e}_{4}\right),
$$

where $z_{i}(i=1,2,3,4)$ are the tracking error vectors defined in Section 3.1 and 3.2. $\boldsymbol{e}_{4}$ is the estimation error $\boldsymbol{e}_{4}=\left[\begin{array}{c}\xi-\hat{\xi} \\ v_{w}-\hat{\boldsymbol{v}}_{w}\end{array}\right], \boldsymbol{P}$ and $\boldsymbol{P}_{\mathrm{e}}$ are positive definite for sufficiently small values of $\varepsilon$. The Lyapunov function in (71) is chosen for the high-level controller as follows:

$$
V_{h} \triangleq \frac{1}{2} \boldsymbol{z}_{4}^{T} P \boldsymbol{z}_{4}+\frac{1}{2} \boldsymbol{e}_{4}^{T} P_{e} \boldsymbol{e}_{4},
$$

where 


$$
P=\left(\begin{array}{cc}
1 & \frac{\varepsilon}{\sigma_{1} \sigma_{2}} \\
\frac{\varepsilon}{\sigma_{1} \sigma_{2}} & \frac{1}{\sigma_{1} \sigma_{2}}
\end{array}\right)
$$

Then the derivative with respect to time of (72) can be obtained by using (66)

$$
\dot{V}_{h}=\frac{1}{2} \boldsymbol{z}_{4}^{T}\left[\left(\begin{array}{cc}
0 & -\sigma_{1} \sigma_{2} \\
1 & -\sigma_{2}
\end{array}\right) P+P\left(\begin{array}{cc}
0 & 1 \\
-\sigma_{1} \sigma_{2} & -\sigma_{2}
\end{array}\right)\right] \boldsymbol{z}_{4}-\boldsymbol{e}_{4}^{T} Q_{e} \boldsymbol{e}_{4} \triangleq-\boldsymbol{z}_{4}^{T} \bar{Q} z_{4}-\tilde{\xi}^{T} Q_{\xi} \tilde{\xi}-\tilde{\boldsymbol{v}}_{w}^{T} Q_{\omega_{w}} \tilde{\boldsymbol{v}}_{w} \leq 0
$$

where $\tilde{\xi}=\xi-\hat{\xi}, \tilde{v}_{w}=v_{w}-\hat{v}_{w}, \bar{Q}=\left(\begin{array}{cc}\varepsilon & \frac{\varepsilon}{2 \sigma_{1}} \\ \frac{\varepsilon}{2 \sigma_{1}} & \frac{\sigma_{2}-\varepsilon}{\sigma_{1} \sigma_{2}}\end{array}\right)$. Substituting (50), (54) and (74) into the derivative of (71) yields

$$
\begin{aligned}
\dot{V} & =-Z_{12}^{T} Q Z_{12}-h \varsigma|\boldsymbol{s}|+\boldsymbol{z}_{3}^{T}\left(\dot{\boldsymbol{v}}-\dot{\boldsymbol{v}}_{d}\right)-\boldsymbol{z}_{4}^{T} \bar{Q}_{4}-\boldsymbol{e}_{4}^{T} Q_{e} \boldsymbol{e}_{4} \\
& =\left(-Z_{12}^{T} Q Z_{12}-\boldsymbol{z}_{3}^{T} K_{3} \boldsymbol{z}_{3}-\boldsymbol{z}_{4}^{T} \bar{Q}_{\boldsymbol{z}_{4}}-\tilde{\xi}^{T} Q_{\xi} \tilde{\xi}-\tilde{v}_{w}{ }^{T} Q_{v_{w}} \tilde{v}_{w}\right)-h \varsigma|\boldsymbol{s}| \\
& \triangleq-\boldsymbol{z}^{T} \Lambda \boldsymbol{z}-\tilde{\xi}^{T} Q_{\xi} \tilde{\xi}-\tilde{v}_{w}{ }^{T} Q_{v_{w}} \tilde{v}_{w}-h \varsigma|\boldsymbol{s}| \\
& \leq-\boldsymbol{z}^{T} \Lambda \boldsymbol{z}-\tilde{\xi}^{T} Q_{\xi} \tilde{\xi}-\tilde{v}_{w}{ }^{T} Q_{v_{w}} \tilde{v}_{w} \\
& \leq-\lambda_{\min }(\Lambda) \boldsymbol{z}^{T} \boldsymbol{z}-\tilde{\xi}^{T} Q_{\xi} \tilde{\xi}-\tilde{v}_{w}{ }^{T} Q_{v_{w}} \tilde{v}_{w}
\end{aligned}
$$

where $\Lambda=\operatorname{diag}\left(Q, K_{3}, \bar{Q}\right), \lambda_{\min }(\boldsymbol{\Lambda})$ is the minimum eigenvalue of $\boldsymbol{\Lambda}$, and $\boldsymbol{z}=\left[\begin{array}{llll}\boldsymbol{z}_{1}^{T} & \boldsymbol{z}_{2}^{T} & \boldsymbol{z}_{3}^{T} & \boldsymbol{z}_{4}^{T}\end{array}\right]^{T}$. For sufficiently small value of $\varepsilon$ meeting with $\min \left\{\sqrt{\sigma_{1} \sigma_{2}}, 4 \sigma_{1} \sigma_{2} /\left(4 \sigma_{1}+\sigma_{2}\right)\right\}$, then $\bar{Q}>0, K_{3}>0$, as $Q_{\xi}>0$ and $Q_{v_{w}}>0$, so $\Lambda$ is positive definite and $\dot{V}$ is negative definite. According to Lemma 1, the system is globally asymptotically stable at $\boldsymbol{z}=\mathbf{0}$. Thus, the reference trajectory $\xi_{r}$ is precisely tracked with $\lim _{t \rightarrow \infty}\left\|\xi(t)-\xi_{r}(t)\right\|=0$.

\subsection{Controllability of the underactuated system analysis}

For the underactuated system, there is no direct control input for one of more degree of freedom, thus the associated oscillatory responses of lateral motion and roll motion will be attenuated by their damping forces. 
Usually the roll damping moment coefficient of $C_{\mathrm{lp}}$ is so large that the corresponding roll moment makes the roll motion convergent quickly [23]. However, this underactuated system without control will be unstable when some adverse weathers are met. Fortunately this underactuated system can be stabilized by coupled moments from other channels. It can be seen from Eq. (63), the control force or moments are determined by their accelerations $\dot{V}=\left[\dot{u}, \dot{v}_{m}, \dot{w}, \dot{p}, \dot{q}, \dot{r}\right]^{T}$ for Case 1 , and $\dot{V}=\left[\dot{u}, \dot{v}_{m}, \dot{w}, \dot{p}_{m}, \dot{q}, \dot{r}\right]^{T}$ for Case 2. According to Eq.(61) and Eq.(62), the virtual acceleration of $\dot{v}_{m}$ and $\dot{p}_{m}$ are related to the yaw acceleration $\dot{r}$ and non-control force of $\left(f_{k y}, f_{w y}, f_{G B y}, f_{A y}\right)$ or non-control moment of $\left(n_{k x}, n_{w x}, n_{G B x}, n_{A x}\right)$. Meanwhile, the yaw acceleration can be obtained from Eq.(4),

$$
\dot{r} \approx \frac{1}{J_{z}}\left(\mathbf{n}_{k z}+\mathbf{n}_{w z}+\mathbf{n}_{G B z}+\mathbf{n}_{A z}\right)+\frac{1}{J_{z}} \mathbf{n}_{C P z}
$$

where $\mathbf{n}_{C P z}=C_{n \delta_{r}} \delta_{r} \bar{q} S_{r e f} l_{r e f}$.

Therefore, the coupled moment resulted from other channel can compensate the underactuate control input. However, since the rudder is mainly for control yaw motion, the roll moment resulted by the simultaneous deflection of the rudder is less than the yaw moment. If a large roll moment input is required to balance the underactuated roll motion, then a large deflection of the rudder is needed which can easily cause the rudder saturation. Therefore, the controllability of the underactuated system is constrained by the coupled channel moment or the coupled control surface deflection.

\section{Simulation and Analysis}

The structure parameters and aerodynamic coefficients of the stratospheric airship are listed in Table 1[24].

Table 1 Parameters and coefficients for the studied airship

\begin{tabular}{ccccc}
\hline \hline Parameter & Value & Unit & Coefficient & Value \\
\hline $\mathrm{m}$ & $5.3 \times 10^{4}$ & $\mathrm{~kg}$ & $k_{1}$ & 0.1054 \\
$\rho$ & 0.072 & $\mathrm{~kg} / \mathrm{m}^{3}$ & $k_{2}$ & 0.8259 \\
$\nabla$ & $7.4 \times 10^{5}$ & $\mathrm{~m}^{3}$ & $k_{3}$ & 0.1247 \\
$x_{G}, z_{G}$ & 0,20 & $\mathrm{~m}$ & $C_{\mathrm{z}}$ & -657 \\
$\mathrm{dx}, \mathrm{dz}$ & 5,5 & $\mathrm{~m}$ & $C_{1}$ & $2.4 \times 10^{4}$ \\
$I_{\mathrm{x}}$ & $4 \times 10^{7}$ & $\mathrm{~kg} \cdot \mathrm{m}^{2}$ & $C_{\mathrm{m}}$ & $-7.7 \times 10^{4}$
\end{tabular}




\begin{tabular}{ccccc}
$I_{\mathrm{y}}$ & $2.3 \times 10^{8}$ & $\mathrm{~kg} \cdot \mathrm{m}^{2}$ & $C_{\mathrm{n}}$ & $-7.7 \times 10^{4}$ \\
$I_{\mathrm{z}}$ & $2.2 \times 10^{8}$ & $\mathrm{~kg} \cdot \mathrm{m}^{2}$ & $k_{1}$ & 0.1054 \\
$I_{\mathrm{xz}}$ & $-4.8 \times 10^{6}$ & $\mathrm{~kg} \cdot \mathrm{m}^{2}$ & & \\
$\mathrm{~m}$ & $5.3 \times 10^{4}$ & $\mathrm{~kg}$ & & \\
\hline
\end{tabular}

The controller parameters for Case 1 are designed as follows

$$
\begin{aligned}
& \sigma_{1}=0.1\left[\frac{1}{\mathrm{~s}}\right], \sigma_{2}=0.2\left[\frac{1}{\mathrm{~s}}\right], c_{\phi}=1\left[\frac{\mathrm{deg} \cdot \mathrm{s}}{\mathrm{m}}\right], c_{\psi}=12\left[\frac{\mathrm{deg} \cdot \mathrm{s}}{\mathrm{m}}\right], \\
& K_{1}=0.5 I_{3}, \lambda_{1}=0.5 I_{3}, K_{3}=4 I_{2}, \gamma=1 \times 10^{6}, W_{v}=I_{5}, W_{u}=I_{6}, \\
& \gamma_{d}=0.2, h=1, \varsigma=1, \zeta=0.4, \varepsilon_{0} \rightarrow 0
\end{aligned}
$$

where $K_{1}, \lambda_{1}$ and $K_{3}$ are chosen to meet the requirements 1) and 2) in Section 2 through iterative design. $I_{\mathrm{i}}$ denotes the $i \times i$ unit matrix, $i=2,3,5,6$.

The position range for the actuators is $\left[-25^{\circ}, 25^{\circ}\right]$, and their rate is confined in $\left[-80^{\circ} / \mathrm{s}, 80^{\circ} / \mathrm{s}\right]$. The initial position is $\xi_{0}=[0,0,-20000 \mathrm{~m}]^{\mathrm{T}}$, the initial body velocity is $\boldsymbol{v}_{0}=[18 \mathrm{~m} / \mathrm{s}, 0,0]^{\mathrm{T}}$, the initial attitude is $\eta_{0}$ $=[0,0,0]^{\mathrm{T}}$, and the initial angular velocity is $\omega_{0}=[0,0,0]^{\mathrm{T}}$.

To validate the trajectory tracking control performance of the ASMB-TTC, a helix reference trajectory function is defined as

$$
\xi_{r}(t)=\left[x_{r}, y_{r}, z_{r}\right]^{T} \triangleq \int_{0}^{t} \dot{\xi}_{r}(t) d \tau+\xi_{r 0},
$$

where the reference velocity $\dot{\xi}_{r}(t)=v_{r}=\left[V_{h} \cos \left(\frac{2 \pi}{T} t\right), V_{h} \sin \left(\frac{2 \pi}{T} t\right),-V_{z}\right]^{T}$ and its initial reference position is $\xi_{r 0}=[40 m,-50 m,-19960 m]^{T}$. The horizontal tracking speed is $V_{h}=20 \mathrm{~m} / \mathrm{s}$, the ascending speed is $V_{z}=1 \mathrm{~m} / \mathrm{s}$, and the motion period is $T=300 \mathrm{~s}$.

Scenario I: Case 1. Trajectory tracking control under small winds.

In this case the wind vector is initially set as $v_{w}=[3,2,1]^{T}(\mathrm{~m} / \mathrm{s})$ during $t<40(\mathrm{~s})$, and is changed into $v_{w}=[1,3,0]^{T}(\mathrm{~m} / \mathrm{s})$ for $t>40(\mathrm{~s})$.

To illustrate our method in attitude control, a backstepping control design by Azinheira [10] is used to compare. In [10], the backstepping control is designed as 


$$
a \dot{y}+\ddot{y}=-\Lambda(a y+2 \dot{y})+\left(a I_{3}+2 \Lambda\right) \tilde{\dot{\eta}}_{w}
$$

where $y=\eta-\eta_{d}, \tilde{\eta}_{w}=\dot{\eta}_{w}-\hat{\dot{\eta}}_{w}, \eta_{w}=0$ denotes attitude variation induced by wind, $\Lambda$ is a diagonal positive-definite matrix used as a tuning parameter, $a$ denotes a scalar tuning parameter.

Then

$$
\ddot{\eta}=\ddot{\eta}_{d}-a \Lambda\left(\eta-\eta_{d}\right)-\left(2 \Lambda+a I_{3}\right)\left(J \omega-\dot{\eta}_{d}\right)+\left(a I_{3}+2 \Lambda\right) \tilde{\dot{\eta}}_{w},
$$

so the desired angular acceleration is obtained as follows

$$
\dot{\omega}=J^{-1}\left[-a \Lambda\left(\eta-\eta_{d}\right)-\left(2 \Lambda+a I_{3}\right)\left(J \omega-\dot{\eta}_{d}\right)+\left(a I_{3}+2 \Lambda\right) \tilde{\dot{\eta}}_{w}-\dot{J} \omega+\ddot{\eta}_{d}\right]
$$

The control parameters in (81) are designed as $a=1, \Lambda=0.5 I_{3}$. Wind parameters in (68) and (69) are chosen as $L_{\xi}=\operatorname{diag}(1,1,1), L_{w}=\operatorname{diag}(0.1,0.1,0.1), Q_{\xi}=I_{3}, Q_{v_{w}}=I_{3}$. Meanwhile the parameters in the guidance loop are chosen as (77). The simulation results are shown in Fig.5-8.
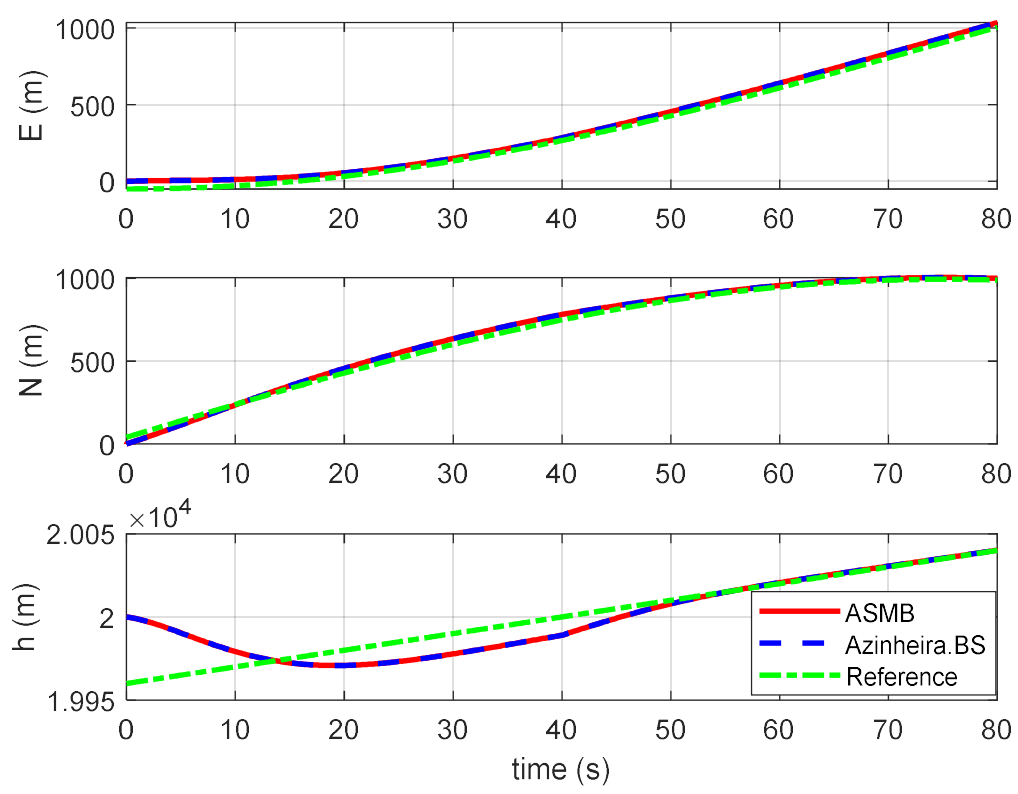

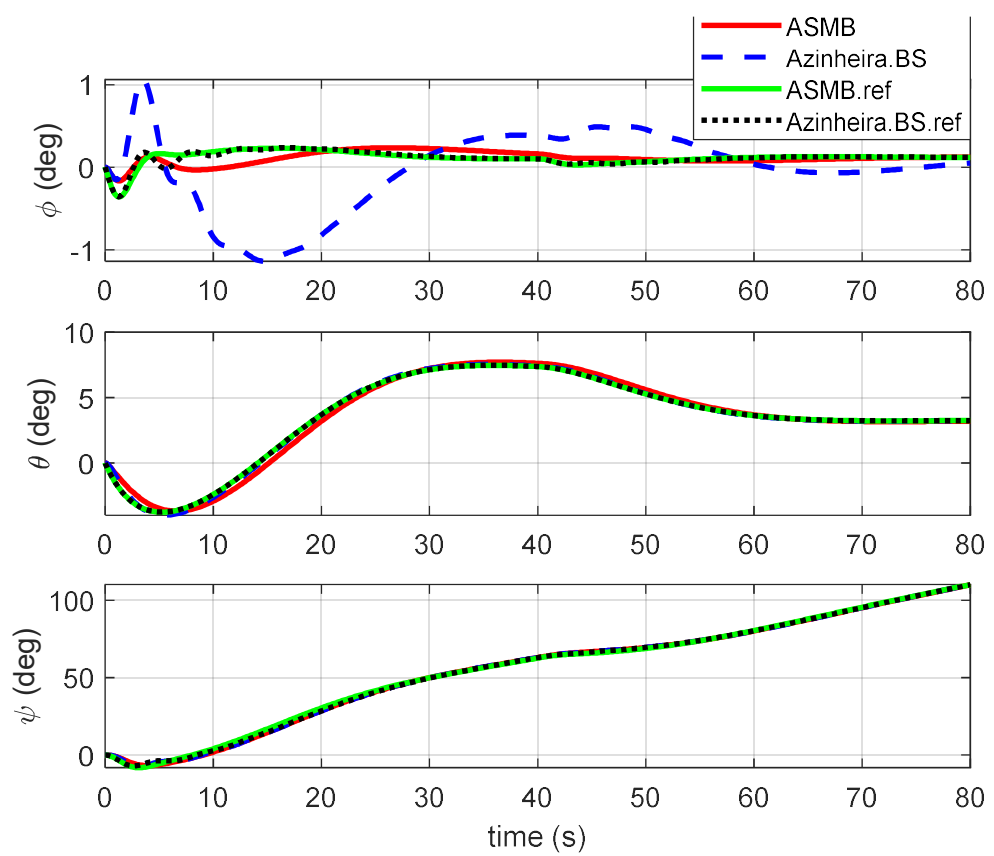

Fig. 5. Position variables and roll-pitch-yaw Euler angles for Case1 with wind inputs
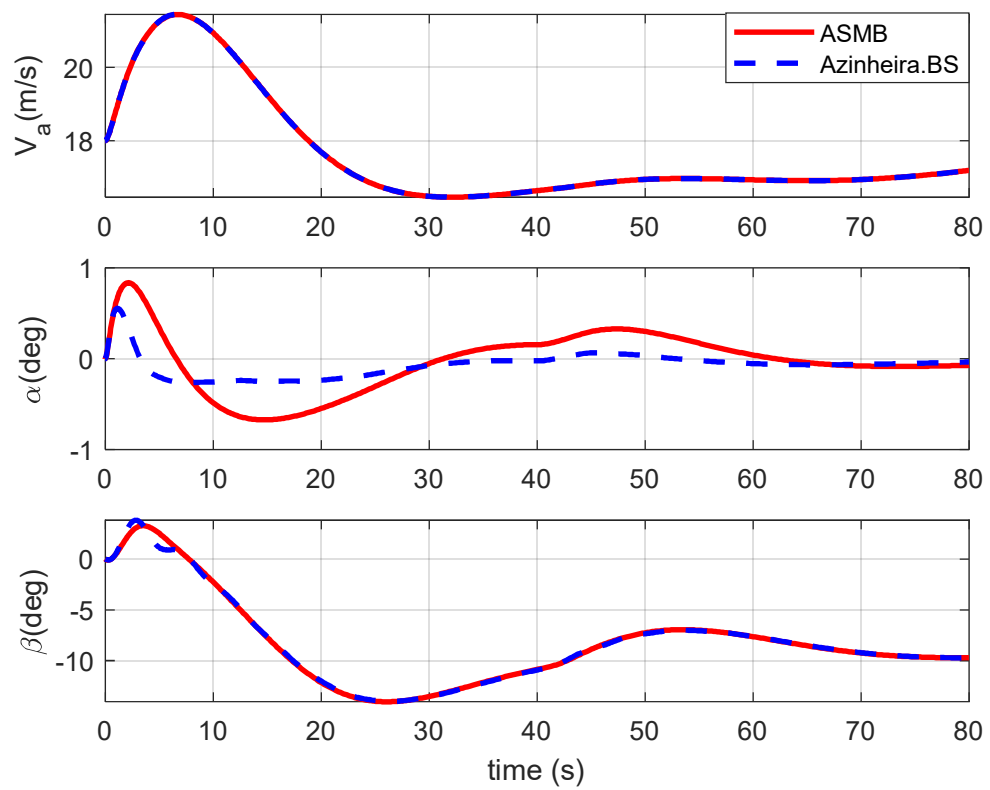

Fig. 6. Airspeed $\left(\mathrm{V}_{\mathrm{a}}\right)$, AOA $(\alpha)$ and sideslip angle $(\beta)$ for Case1 with wind inputs 

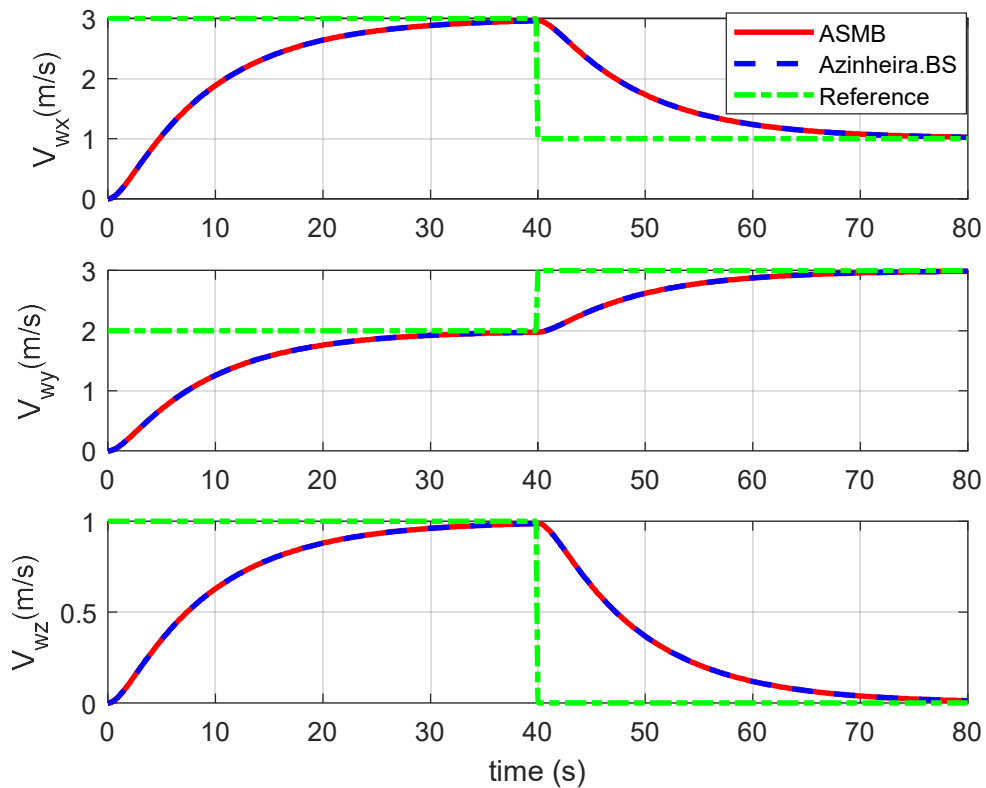

Fig. 7. Wind inputs and their estimation for Case 1
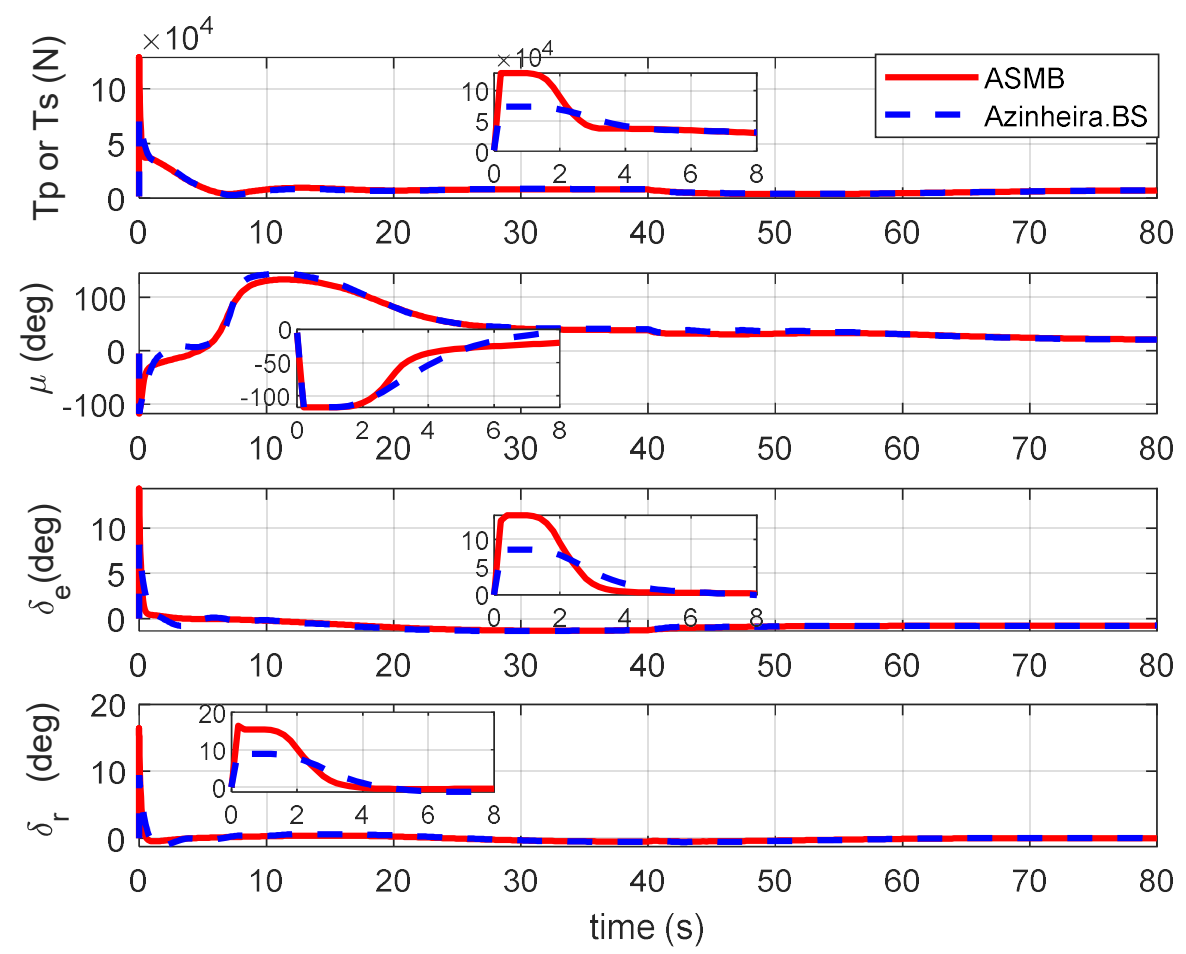

Fig. 8. Control inputs for Case 1 with wind 
From Fig.5, it is clear that the reference trajectory and attitudes have been accurately tracked by using ASMB control and the tracking errors converge to the desired position within 50 seconds, even in presence of the wind disturbance. For the response of roll motion there are some oscillations because of weak roll damping of the airship, so they need more regulating time for convergence. Comparing with Azinheira's Backsptepping control, the proposed ASMB control has less tracking errors in roll angle motion, which shows ASMB has more capability to deal with the under actuation of the airship. In Fig.6 the tracking response of airspeed $\left(V_{T}\right)$ has a fast convergence with small oscillation, and the AOA $(\alpha)$ response of ASMB has more overshoot than that of Azinheira's Backsptepping design due to wind disturbances, also for the sliding angle $(\beta)$ response. Fig.7 shows the wind vector can be accurately estimated by the wind observer. Here the deflections of the left- and right- elevator are equal half of the total elevator deflection, see Fig.8. Similarly for the upper and lower rudders. From Fig. 8 it can be seen that the control surface inputs and the thruster of ASMB are greater than Azinheira's BS control, but the tilt angle of the propellers is less than those of Azinheira's BS control. Furthermore Azinheira's BS control is easy affected by the wind speed (changing at the time 40s). This demonstrates the sliding mode term in the backstepping design improves the system adaptive performance.

Scenario II: Case 2 Trajectory tracking control under parameter uncertainty

This scenario considers model parameter uncertainty having the aerodynamic derivatives vary with angle of attack (AOA). So aerodynamic coefficients are set as follows

$$
\left[\begin{array}{c}
C_{x t} \\
C_{y t} \\
C_{z t} \\
C_{m x t} \\
C_{m y t} \\
C_{m z t}
\end{array}\right]=\left[\begin{array}{c}
(1.2+0.5 \alpha) C_{x} \\
(1.2+0.5 \alpha) C_{y} \\
(1.2-0.5 \alpha) C_{z} \\
(0.8-0.5 \alpha) C_{m x} \\
(0.8-0.5 \alpha) C_{m y} \\
(0.8-0.5 \alpha) C_{m z}
\end{array}\right]
$$

where a subscript of $t$ denotes perturbation value. In this scenario the parameter uncertainty and external wind disturbances are considered simultaneously. 
The control parameters are designed as follows,

$$
\begin{aligned}
& \sigma_{1}=0.1\left[\frac{1}{\mathrm{~s}}\right], \sigma_{2}=0.2\left[\frac{1}{\mathrm{~s}}\right], c_{\psi}=12\left[\frac{\mathrm{deg} \cdot \mathrm{s}}{\mathrm{m}}\right], \\
& K_{1}=\lambda_{1}=0.5 I_{2}, K_{3}=4 I_{2}, \gamma=1 \times 10^{6}, W_{v}=I_{4}, \quad(\text { Case 2) } \\
& W_{u}=I_{4}, \gamma_{d}=0.2, h=\varsigma=1, \zeta=0.4, \varepsilon_{0} \rightarrow 0
\end{aligned}
$$

The reference trajectory function of (78) is the same as Case 1 . To compare control performances, Scenario II is simulated by using the PID controller with control parameters of $K_{P}=4, K_{I}=0.0$ and $K_{D}=0.08$ in the three channels of roll, pitch and yaw. Meanwhile the parameters in the guidance loop are chosen as (77). The 3-D reference trajectory and its trajectory tracking responses are shown in Fig.9-14. It can be seen that desired positions are well tracked, but the roll responses are fluctuating with a small amplitude. The reason is the underactuation in the roll motion of the airship.
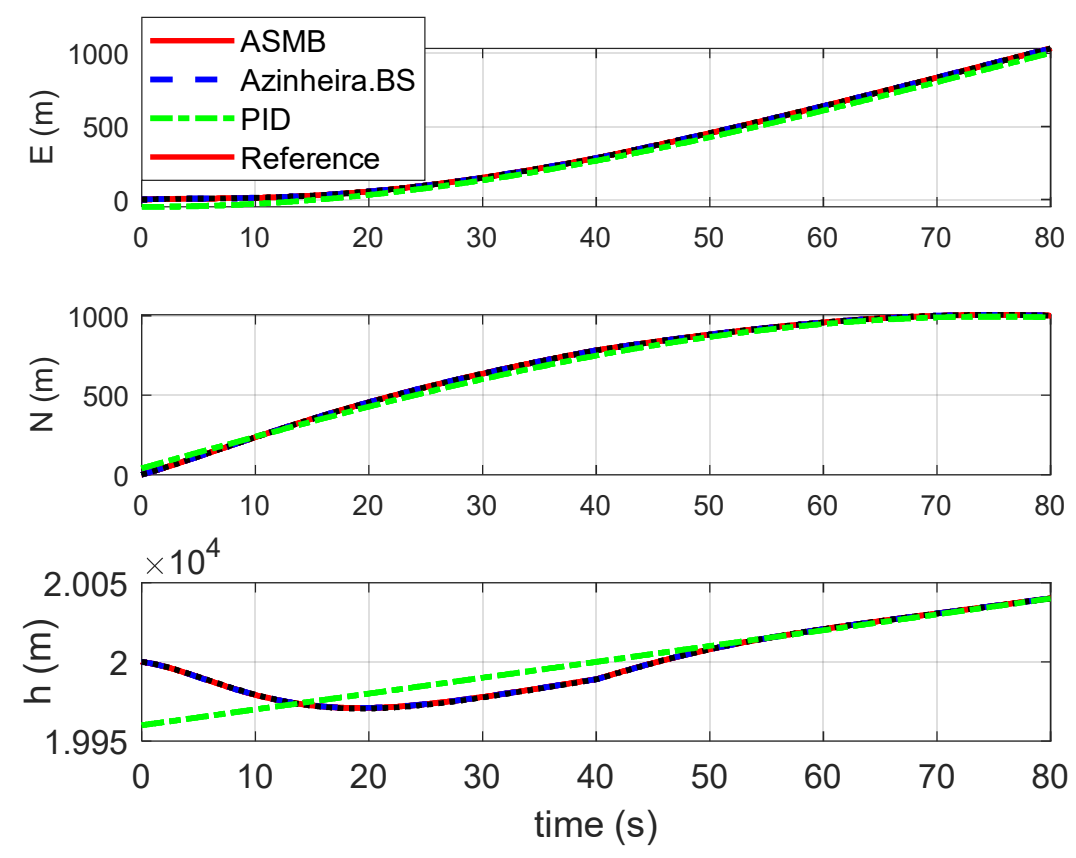

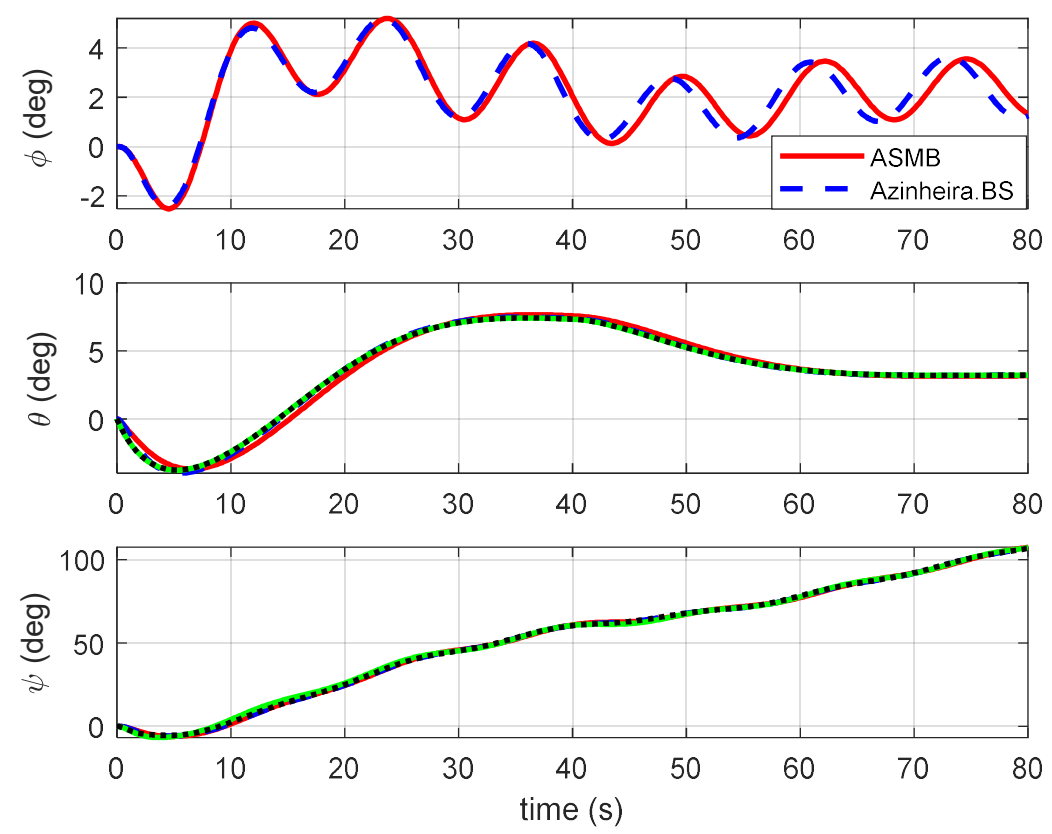

Fig.9. Positions and Euler angles for Case 2 with model uncertainty and variable winds

From Fig.9 it can be seen that responses of attitude angles for Azinheira's BS control have large overshoot and tracking errors than those for the ASMB method, which shows the ASMB design has more adaptive capability for aerodynamic coefficient uncertainty.
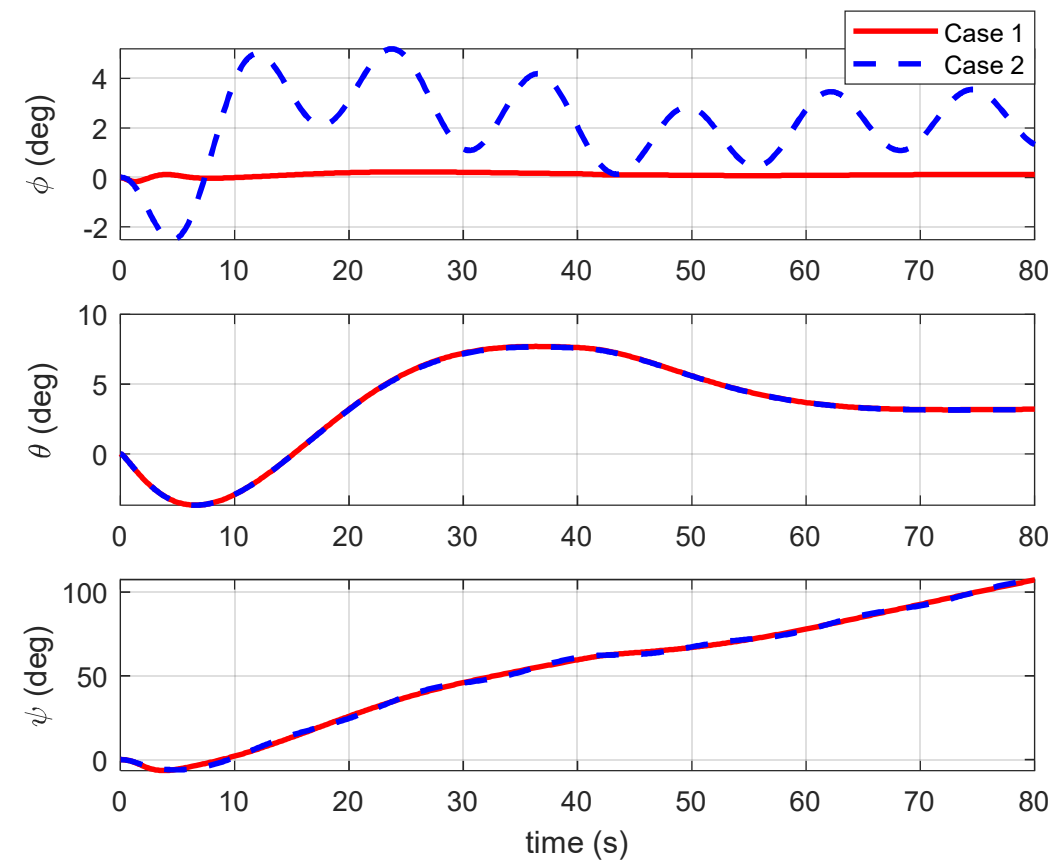

Fig. 10. Euler angles of ASMB control for Case1 and Case 2. 
Comparing Case 1 with Case 2 in roll responses, it can be seen from Fig. 10 that the bank angle $\phi$ in Case 1 is smaller than that in Case 2. The reason is that the roll moment is actuated in Case 1 while it is underactuated in Case2, the desired pitch and bank angles can be tracked with good performance.

From Fig. 6 and 11 it is observed that sideslip angles of both cases are bounded, which implies that the sway velocities of both cases are bounded. The simulation results are in accordance with Theorem 1 and Remark 5 .
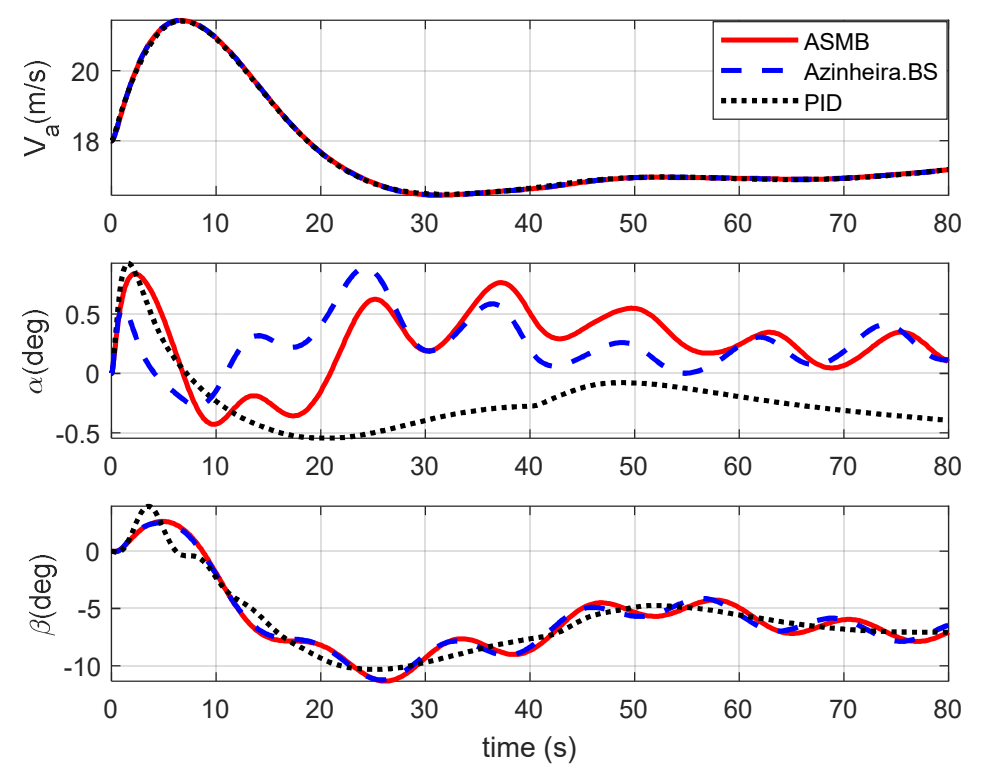

Fig.11. Airspeed, AOA and side-sliding angle for Case2 with model uncertainty and disturbances.

Fig.11 shows the PID control is more affected by the parameter uncertainty, and ASMB control is robust and adaptive for this uncertainty. The control inputs are shown in Fig.12, from which it can be seen that the responses of the control input are oscillating for more than $80 \mathrm{~s}$ because of the roll underactuation. The rudder $\delta_{\mathrm{r}}$ and propeller tilt angle using Azinheira's BS control are more than those using ASMB and PID in transient time, but the elevator $\delta_{\mathrm{e}}$ and thruster input of Azinheira's BS control are less than those of the ASMB, and PID controller. This shows that the ASMB control makes the response converge faster by using large thruster inputs. Azinheira's BS control needs more input of the rudder and propeller tilt angle to stabilize for model parameter uncertainty, especially for the wind changing at the time of 40s. Thus it shows ASMB has more 
capability to reject model parameter uncertainty.
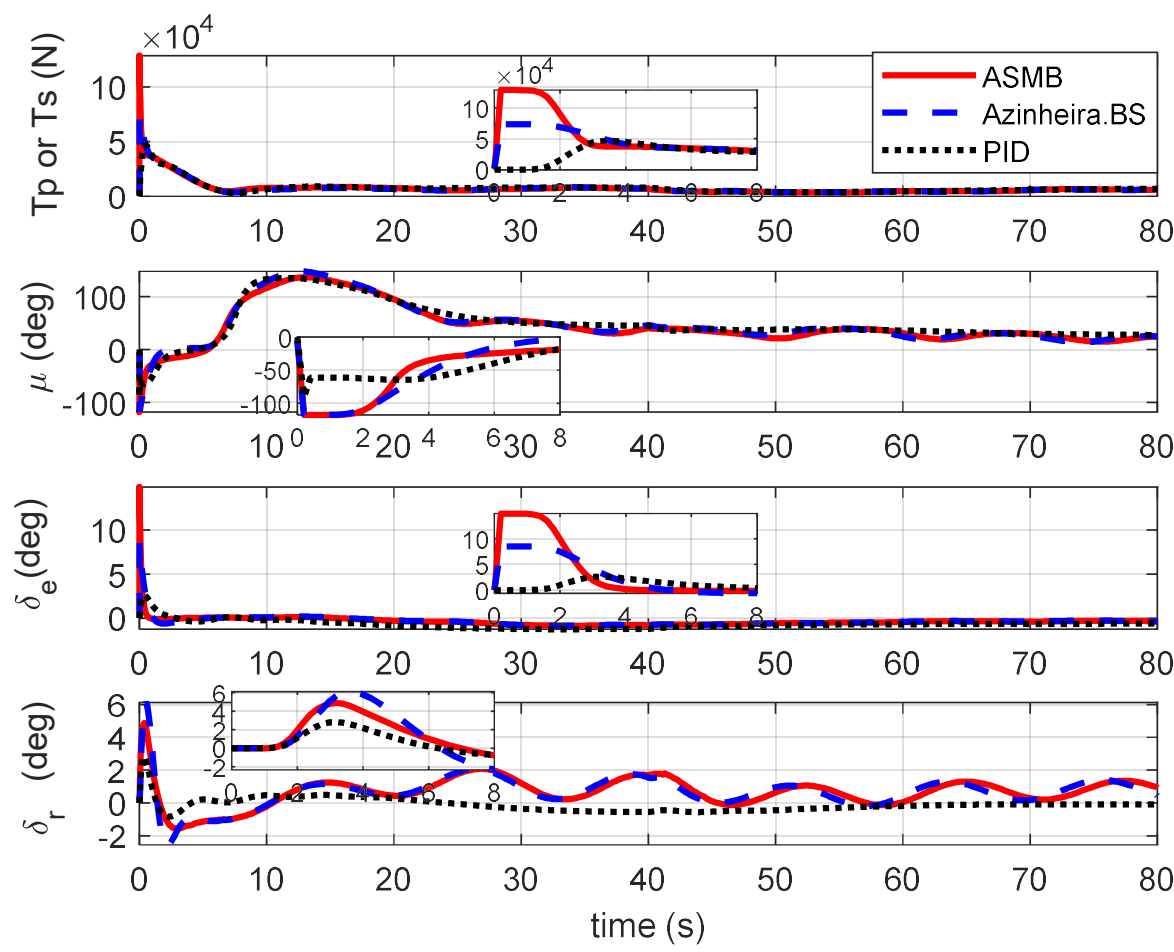

Fig. 12. Control inputs for Case 2 with model uncertainty and external disturbances.
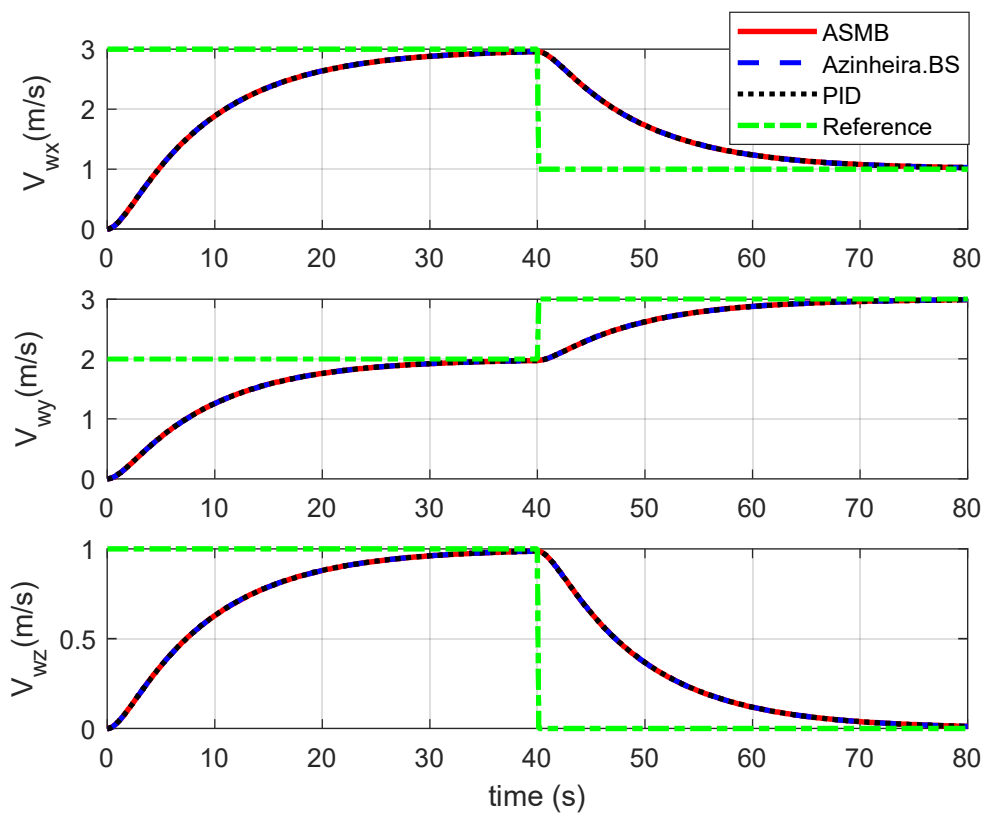

Fig.13. Estimation of wind speed 

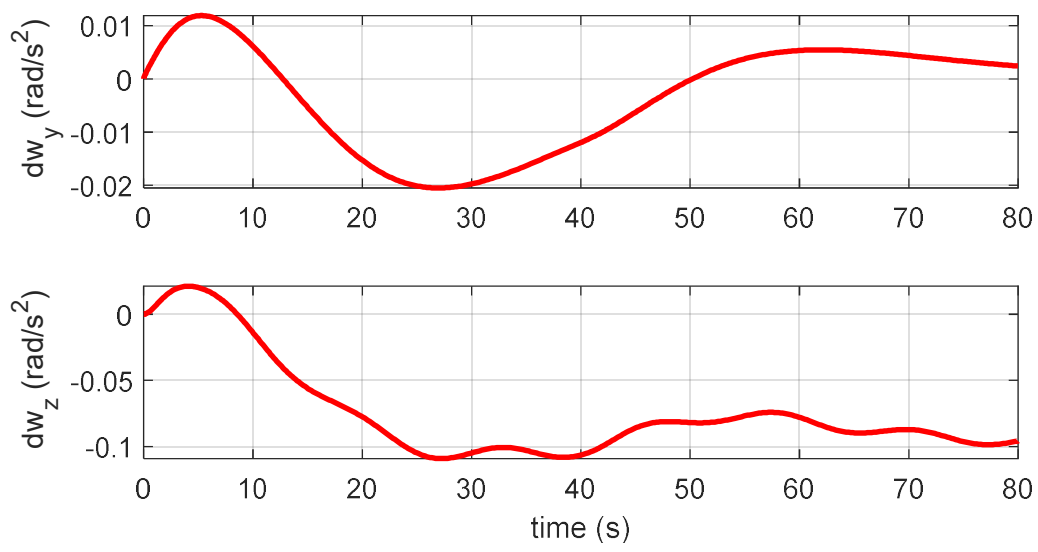

Fig. 14. Estimation of the attitude disturbance in Case 2.

The wind and aerodynamic coefficient uncertainty disturbances in the pitch and yaw directions as (38) are estimated, the results are shown in Fig.13 and 14.

Scenario III: Case $1 \& 2$ controllability of the underactuated system under large wind disturbances

First the coupled channel control compensation is studied for Case 1 . The wind vector is initially set as $v_{w}=[3,12,1]^{T}(\mathrm{~m} / \mathrm{s})$ for $t<40(\mathrm{~s})$, and is changed to $v_{w}=[1,2,0]^{T}(\mathrm{~m} / \mathrm{s})$ for $t>40(\mathrm{~s})$. When there is no coupled channel control compensation for roll motion, a roll damping moment is used to control the roll motion with a damping coefficient $C_{l p}=-C_{D c g} S_{g} l_{g z} \sin (\beta) \sin (\operatorname{abs}(\beta))^{[24]}$. By using the proposed ASMB control, the results are shown in Fig.15 and 16. 

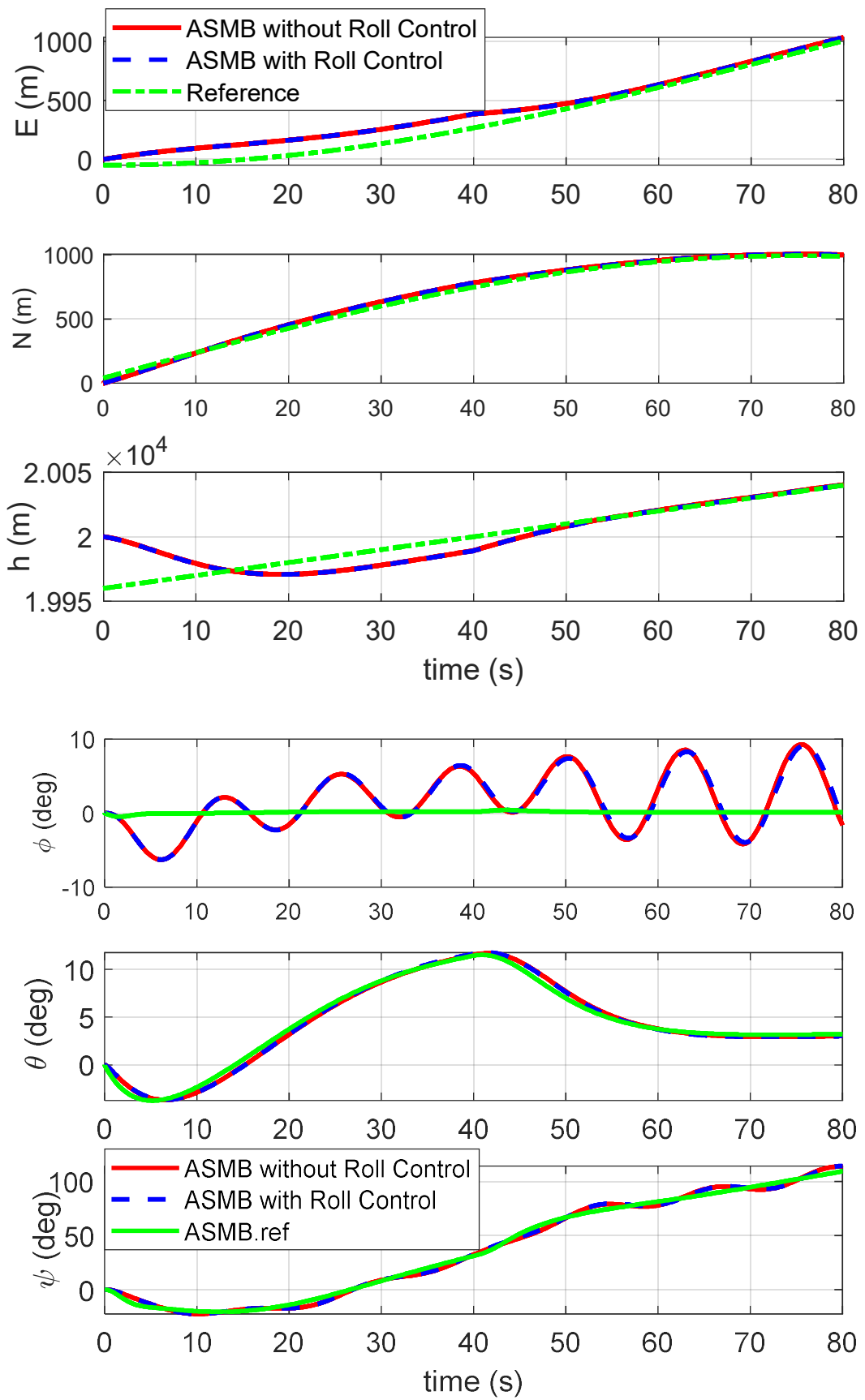

Fig. 15. Position variables and Euler angles for Case1 with wind inputs 

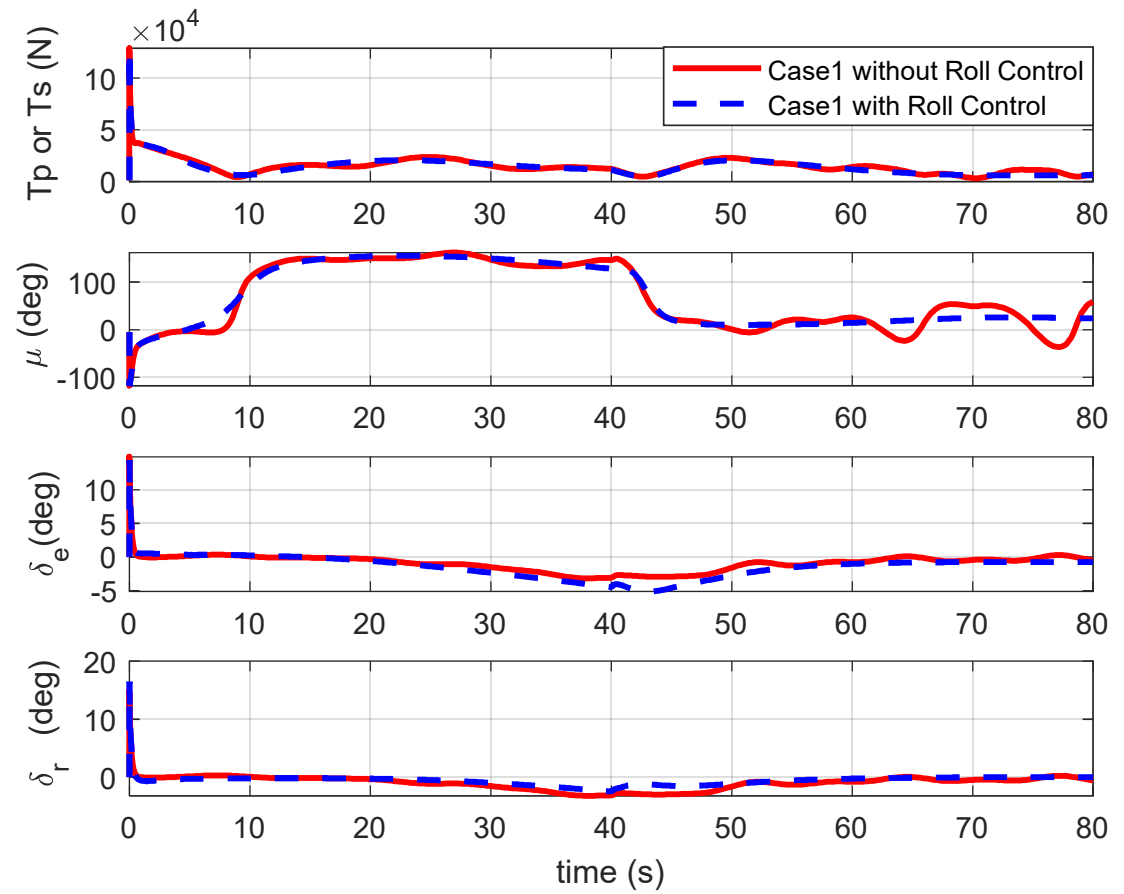

Fig. 16. Control inputs for Case 1 with and without coupled compensation

Fig. 15 shows that subject to wind with lateral speed $v_{w y}=12 \mathrm{~m} / \mathrm{s}$, the east position has large tracking errors whether there is coupled channel compensation or not. Meanwhile, the roll angle responses oscillate with the increase of wind speed. From the control inputs it can be seen from Fig.16 that the inputs with roll compensation from coupled channels are smaller than those without compensation. This is because the roll coupled channel compensation makes the output roll response converge quickly while the other converges very slowly and only depends on its damping moment.

Second, the large lateral winds are chosen to excite system instability under lateral and roll underactuated cases. The wind vector is initially set $v_{w}=[3,12,1]^{T}(\mathrm{~m} / \mathrm{s})$ for $t<40(\mathrm{~s})$, and is changed to $v_{w}=[1,20,0]^{T}(\mathrm{~m} / \mathrm{s})$ for $t>40(\mathrm{~s})$. The proposed ASMB control results are shown as Fig.17-19. 

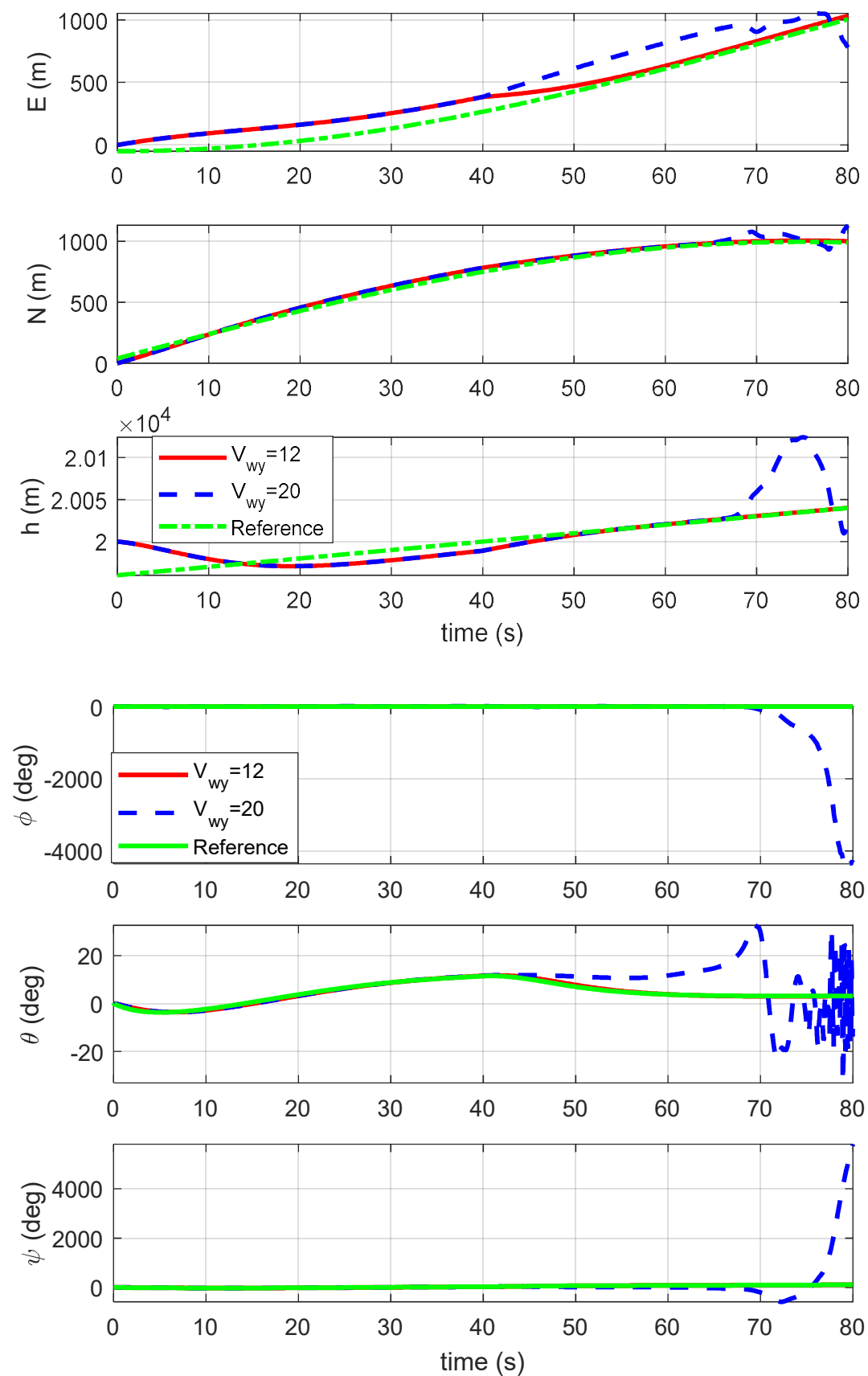

Fig.17. Position variables and Euler angles with different lateral wind speeds 

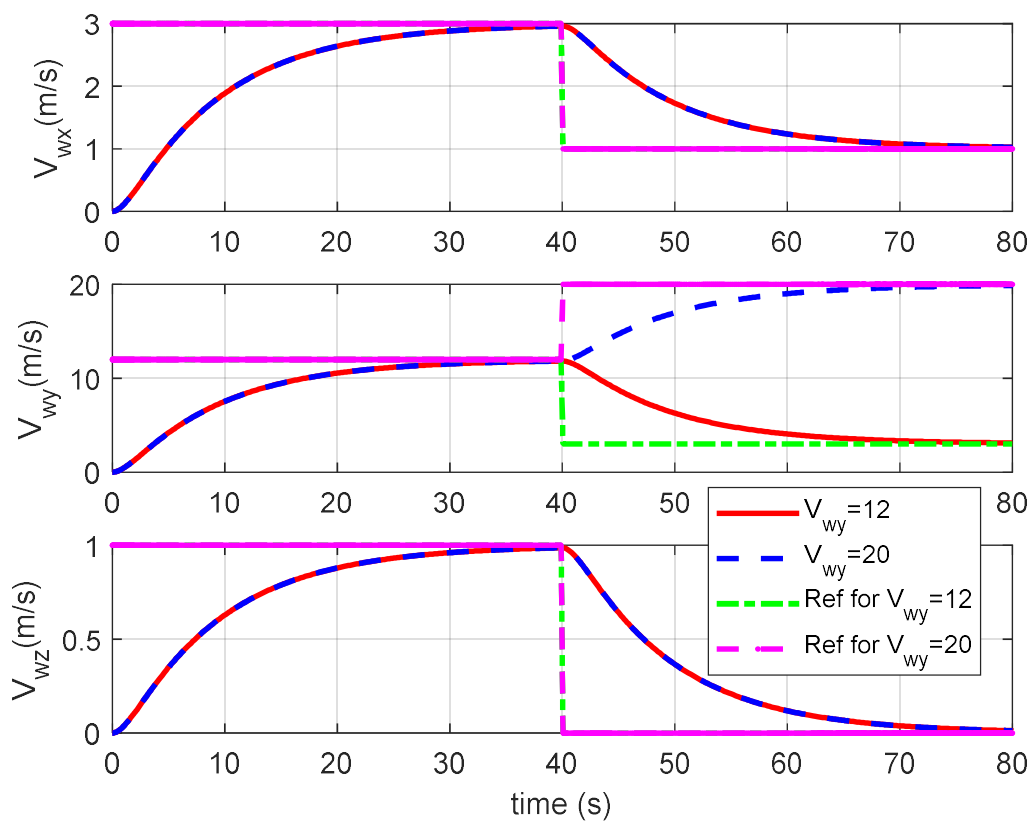

Fig. 18. Wind inputs and their estimation for Case 1 and Case 2
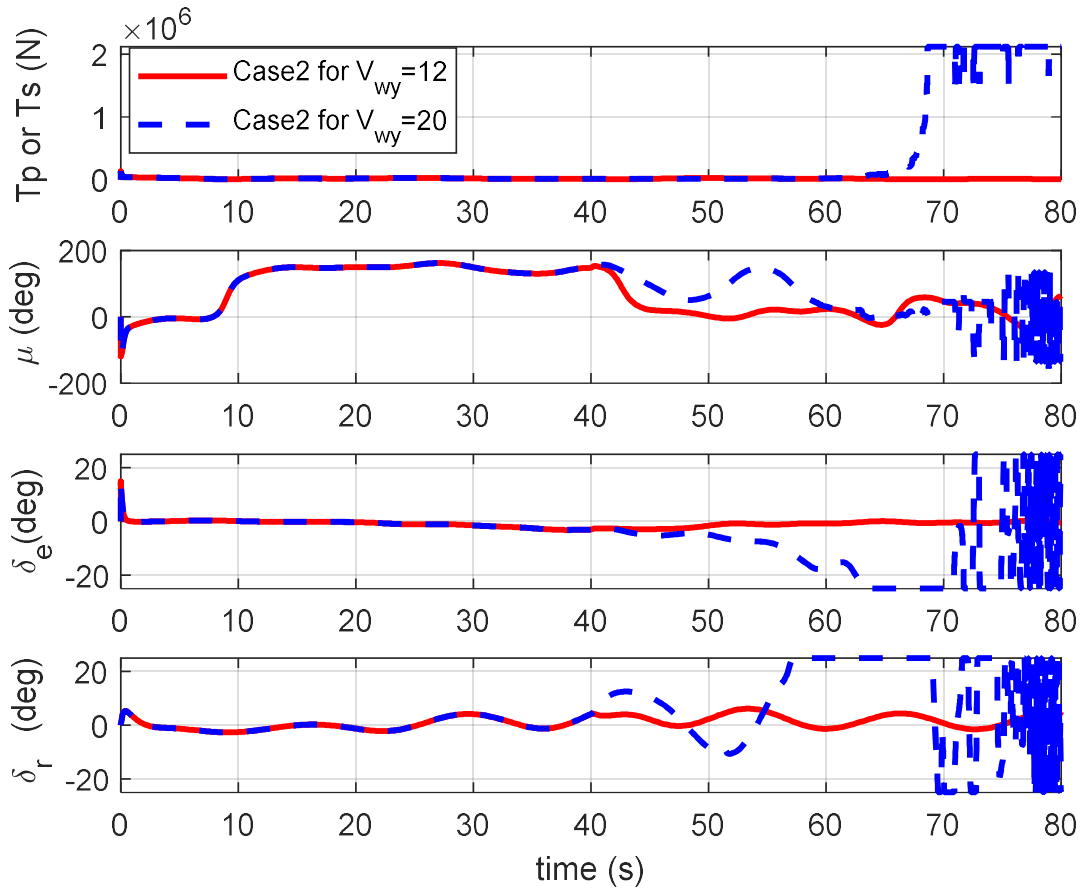

Fig. 19. Control inputs for Case 1 with different wind speeds.

From Fig. 17, it can be seen that the underactuated system becomes unstable when the lateral wind speed increases from $v_{\mathrm{wy}}=12 \mathrm{~m} / \mathrm{s}$ to $v_{\mathrm{wy}}=20 \mathrm{~m} / \mathrm{s}$, the attitude outputs begin to diverge from the references, and the output responses of the position have increasing magnitude oscillations for Case 1 and Case 2. This shows that the large lateral wind speed makes the underactuated system lose control or exceed the controllability for the underactuated airship. The corresponding wind speeds are estimated in Fig.18. Fig.19 shows the control 
inputs with different wind speeds. It can be seen that the control inputs of thrust and control surfaces saturate when the lateral wind speed increases to $v_{\mathrm{wy}}=20 \mathrm{~m} / \mathrm{s}$. So the controllability for the underactuated airship is constrained by the available control inputs.

\section{Conclusion}

In this paper we propose the ASMB-TTC approach for the underactuated stratospheric airship. Based on a full 6-DOF nonlinear model, the trajectory tracking controller is designed. The developed controller stabilizes the attitude and velocity of the airship via the adaptive sliding mode backstepping method. Furthermore, an active set base weighted least square algorithm is applied to find the optimal thruster and control surface inputs under constraints of saturation. Stability analysis shows that the closed-loop trajectory tracking error dynamics are globally exponentially stable. Two cases of the lateral and roll underactuations were simulated and showed the proposed robustness. Compared with a conventional PID controller, the ASMB-TTC achieves better trajectory tracking performances even though the airship is affected by parametric uncertainties and external bounded disturbances. Therefore, the effectiveness and availability of the ASMB-TTC design are demonstrated. The approach could possibly be extended to other airship configurations. For example, airships can be controlled by moving mass technologies [30]. Investigation of the proposed method for such technologies remains for future work [31, 32].

\section{Acknowledgements}

This work has been funded by the Chinese Aviation Science Fund under the Grant 20160757001, and the National Natural Science Fund under the Grant 10577012. The work of Shiqian Liu was supported by the China Scholarship Council under the grant number 201806235014.

\section{Nomenclature}
$\alpha, \beta$
angle of attack, sideslip angle, rad
$B, G$
airship buoyancy, gravity, $\mathrm{N}$
$C_{l}, C_{m}, C_{n}$ aerodynamic moment coefficients along the body axis
$d, d_{V}, d_{\omega}$ model uncertainty or external disturbances, its subvariant of linear and angular acceleration, $\mathrm{m} / \mathrm{s}^{2}, \mathrm{rad} / \mathrm{s}^{2}$ 


$\mathbf{F}_{\mathrm{b}}, \mathbf{F}_{\mathrm{g}}, \mathbf{F}_{\mathrm{w}}$
$\boldsymbol{F}_{A}, \boldsymbol{F}_{C P}, \boldsymbol{F}_{k}$,
$\boldsymbol{F}_{w}, \boldsymbol{F}_{G B}$
$f_{\mathrm{A} i}, f_{\mathrm{CP} i}, f_{\mathrm{k} i}, f_{\mathrm{w} i} f_{\mathrm{GB} i}$
$n_{\mathrm{A} i}, n_{\mathrm{CP} i}, n_{\mathrm{k} i}$,
$n_{\mathrm{w} i}, n_{\mathrm{GB} i}$
$n_{\mathrm{d}}$
$\bar{q}$
$T_{p}, T_{s}$
$\boldsymbol{v}, \boldsymbol{v}_{d}, \boldsymbol{v}_{c}, \boldsymbol{v}_{r}$
$\boldsymbol{v}_{a}, \boldsymbol{v}_{g}, \boldsymbol{v}_{w}$
$\boldsymbol{\omega}$
$\boldsymbol{V}=[\boldsymbol{v}, \boldsymbol{\omega}]^{\mathrm{T}}$
$\boldsymbol{z}_{i}$
$\delta_{e L}, \delta_{e R}$
$\delta r_{U}, \delta r_{B}$
$\mu$
$\boldsymbol{\eta}, \boldsymbol{\eta}_{\boldsymbol{d}}, \boldsymbol{\eta}_{\boldsymbol{r}}$
$\boldsymbol{\xi}, \boldsymbol{\xi}_{\mathrm{r}}$
$\boldsymbol{\xi}_{\boldsymbol{w}}$
$\phi, \theta, \psi$
$\nabla$

the body fixed frame, the Earth reference frame, the wind-axes frame vectors of aerodynamic force, sum of control and thrusts, kinematics force, wind-induced force,sum of gravity and buoyancy respectively, $\mathrm{N}$ forces of $\boldsymbol{F}_{A}, \boldsymbol{F}_{C P}, \boldsymbol{F}_{k}, \boldsymbol{F}_{w}, \boldsymbol{F}_{G B}$ along $i$ axis $\left(i=x_{\mathrm{b}}, y_{\mathrm{b}}, z_{\mathrm{b}}\right), \mathrm{N}$ moments resulted by $\boldsymbol{F}_{A}, \boldsymbol{F}_{C P}, \boldsymbol{F}_{k}, \boldsymbol{F}_{w}, \boldsymbol{F}_{G B}\left(i=x_{\mathrm{b}}, y_{\mathrm{b}}, z_{\mathrm{b}}\right), \mathrm{N} \cdot \mathrm{m}$

desired moment vector, $\mathrm{N} \cdot \mathrm{m}$ dynamic pressure, $\mathrm{Pa}$ thrusts of the port side and the starboard side, $\mathrm{N}$ airship practical, desired, commanded and reference translational velocity vector in $\mathbf{F}_{\mathrm{g}}, \mathrm{m} / \mathrm{s}$ airship airspeed in $\mathrm{F}_{\mathrm{b}}$, ground speed and wind speed in $\mathrm{F}_{\mathrm{g}}$, $\mathrm{m} / \mathrm{s}$

airship angular rate vector $[p, q, r]^{\mathrm{T}}, \mathrm{rad} / \mathrm{s}$ the generalized velocity in $\mathbf{F}_{\mathrm{b}}$ virtual state in the controller design $(i=1,2,3,4)$ left and right elevator deflection, rad upper and bottom rudder deflection, rad tilt angle of the propellers, rad practical attitude vector, desired and reference attitude vectors, $\mathrm{rad}$ practical and reference position vectors $[x, y, z]^{\mathrm{T}}$ in $\mathbf{F}_{g}, \mathrm{~m}$ wind position vectors $\left[x_{w}, y_{w}, z_{w}\right]^{\mathrm{T}}$ in $\mathbf{F}_{g}, \mathrm{~m}$ airship Euler angles: roll, pitch, yaw, rad volume of the airship hull, $\mathrm{m}^{3}$

\section{References}

1. Kulczycki EA, Joshi SS, Hess RA, Elfes A. Towards controller design for autonomous airships using SLC and LQR methods, in: AIAA Guidance, Navigation, and Control Conference and Exhibit, Keystone, CO., August 2006, pp.1-16.

2. Schmidt D. K. Modeling and near-space stationkeeping control of a large high altitude airship. Journal of Guidance, Control, and Dynamics. 30(2) (2007) 540-547.

3. Du H.F., Li J., Zhu W. Y., Qu Z. P., Zhang L. C., et al., Flight performance simulation and station-keeping endurance analysis for stratospheric super-pressure balloon in real wind field. Aerospace Science and Technology. 86 (2019) 1-10. 
4. Moutinho A, Azinheira JR. Stability and robustness analysis of the AURORA airship control system using dynamic inversion, in: Proceedings of the 2005 IEEE International Conference on Robotics and Automation, Barcelona, Spain, April 2005, pp. 2277-2282.

5. Hu J. S., Huang J. Z., Gao Z. X., Gu H.B., Position tracking control of a helicopter in ground effect using nonlinear disturbance observer-based incremental backstepping approach, Aerospace Science and Technology. 81 (2018) 167-178

6. Wang X. Y., Yu X., Li S. H., Liu J. Y., Composite block backstepping trajectory tracking control for disturbed unmanned helicopters, Aerospace Science and Technology. 85 (2019) 386-398

7. Hygounenc E, Soueres P. Automatic airship control involving backstepping techniques, in: IEEE International Conference on Systems, Man and Cybernetics, Yasmine Hammamet, Tunisia, 2002; 1-6.

8. Lee S, Lee H, Won D, Bang H. Backstepping approach of trajectory tracking control for the mid-altitude unmanned airship, in: AIAA Guidance, Navigation and Control Conference and Exhibit, Hilton Head, SC, August 2007,pp. 1-14.

9. Azinheira JR, Moutinho A, de Paiva EC. Airship hover stabilization using a backstepping control approach. Journal of Guidance, Control, and Dynamics. 29(4) (2006) 903-914.

10. Azinheira JR, Moutinho A, de Paiva EC. A backstepping controller for path-tracking of an underactuated autonomous airship. International Journal of Robust and Nonlinear Control. 19(4)(2009) 418-441.

11. Yang Y, Wu J, and Zheng W. Station-keeping control for a stratospheric airship platform via fuzzy adaptive backstepping approach. Advances in Space Research. 51(7) (2013) 1157-1167.

12. Moutinho A, Azinheira JR, de Paiva EC, Bueno SS. Airship robust path tracking: A tutorial on airship modeling and gain scheduling control design. Control Engineering Practice. 50 (2016) 22-36.

13. Liu SQ, Sang YJ, Jin HB. Robust model predictive control for a stratospheric airships using LPV design. Control Engineering Practice. 81 (2018) 231-243. 
14. Repoulias F, Papadopoulos E. Robotic airship trajectory tracking control using a backstepping methodology, in: IEEE International Conference on Robotics and Automation, Pasadena, CA, May 2008, pp. $188-193$.

15. Zheng Z, Wu Z. Global path following control for underactuated stratospheric airship. Advances in Space Research. 52(7) (2013) 1384-1395.

16. Daskiran O, Dogan A. Airship control using expert demonstrations, in: AIAA Atmospheric Flight Mechanics Conference, Washington D. C., USA, June 2016, pp. 1-28.

17. Liesk T, Nahon M, Boulet B. Design and experimental validation of a nonlinear low-level controller for an unmanned fin-less airship. IEEE Transactions on Control Systems Technology. 21(1) (2013) 149-16

18. Liu SQ, Gong SJ, Lu ZR. Vectorial backstepping method based trajectory tracking control for an under-actuated stratospheric airship. The Aeronautical Journal. 121(1241) (2017) 916-938.

19. Yang N., Yan Y., Neural network approximation-based nonsingular terminal sliding mode control for trajectory tracking of robotic airships, Aerospace Science and Technology. 54 (2016) 192-197

20. Azinheira JR, Moutinho A. Hover control of an UAV with backstepping design including input saturations. IEEE Transactions on Control Systems Technology. 16(3) (2008) 517-526.

21. Liesk T, Nahon M, Boulet B. Design and experimental validation of a controller suite for an autonomous finless airship, in: American Control Conference, Fairmont Queen Elizabeth, Montreal, Canada, June 2012, pp. 2491-2496.

22. Gomes SBV. An investigation into the flight dynamics of airships with application to the YEZ-2A. PhD Thesis. Cranfield University, 1990.

23. Khoury GH, Gillett JD. Airship Technology, Chapter 4, Cambridge University Press, Cambridge, U.K., 2012, pp. 60-85. 
24. Mueller JB, Paluszek MA, Zhao Y. Development of an aerodynamic model and control law design for a high altitude airship, in: AIAA 3rd Workshop and Exhibit of "Unmanned Unlimited" Technical Conference, Chicago, IL, September 2004, pp. 1-17.

25.Azinheira JR, de Paiva EC, Bueno SS. Influence of wind speed on airship dynamics. Journal of Guidance, Control, and Dynamics. 25(6) (2002) 1116-1124.

26. Healey AJ. Multivariable sliding mode control for autonomous diving and steering of unmanned underwater vehicles. IEEE Journal of Oceanic Engineering. 18(3) (1993) 327-339.

27. Gao WB. Variable Structure Control Theory and Design Method, Science Publisher ,Beijing, 1996, pp.48.

28. Liesk T, Nahon M, Boulet B. Design and Experimental Validation of a Controller Suite for an Autonomous Finless Airship, in: American Control Conference, Fairmont Queen Elizabeth, Montreeal, Canada, June 2012, pp. 2491-2496.

29. Fossen TI. Guidance and Control of Ocean Vehicles, Appendix C. John Wiley \& Sons LTD, 1994, pp.411-412.

30. Chen L., Zhou G, Yan X.J., Duan D. P., Composite control strategy of stratospheric airships with moving masses, AIAA Journal of Aircraft, 49(3), (2012):794-801.

31. Li J. Q., Gao C. S., Li C. Y., Jing W. X., Fan Y. D., Nonlinear vibration analysis of a novel moving mass flight vehicle, Nonlinear Dynamics 90 ( 2017):733-748

32. Li J. Q., Gao C. S., Li C. Y., Jing W. X., A survey on moving mass control technology, Aerospace Science and Technology, 82-83 (2018) 594-606 
2019-12-12

\title{
Adaptive sliding-mode-backstepping trajectory tracking control of underactuated airships
}

\author{
Liu, Shi Qian
}

Elsevier

Liu SQ, Sang YJ, Whidborne J. (2019) Adaptive sliding-mode-backstepping trajectory tracking control of underactuated airships. Aerospace Science and Technology, Volume 97, February 2020, Article number 105610

https://doi.org/10.1016/j.ast.2019.105610

Downloaded from Cranfield Library Services E-Repository 